This item was submitted to Loughborough's Research Repository by the author.

Items in Figshare are protected by copyright, with all rights reserved, unless otherwise indicated.

\title{
Validation of a large eddy simulation methodology for accelerated nozzle flows
}

PLEASE CITE THE PUBLISHED VERSION

https://doi.org/10.1017/aer.2020.12

\section{PUBLISHER}

Cambridge University Press (CUP)

VERSION

AM (Accepted Manuscript)

\section{PUBLISHER STATEMENT}

This article has been published in a revised form in The Aeronautical Journal https://doi.org/10.1017/aer.2020.12. This version is published under a Creative Commons CC-BY-NC-ND. No commercial re-distribution or re-use allowed. Derivative works cannot be distributed. (๑) The Authors.

\section{LICENCE}

CC BY-NC-ND 4.0

\section{REPOSITORY RECORD}

Wang, PC, and Jim McGuirk. 2020. "Validation of a Large Eddy Simulation Methodology for Accelerated Nozzle Flows". Loughborough University. https://hdl.handle.net/2134/14053895.v1. 


\title{
Validation of a Large Eddy Simulation Methodology for Accelerated Nozzle Flows
}

\author{
P. C. Wang ${ }^{1} \&$ J. J. McGuirk. \\ Dept of Aero \& Auto Eng., \\ Loughborough University, \\ Loughborough LE11 3TU, UK.
}

\begin{abstract}
Prediction of aeroengine exhaust plume near-field development requires knowledge of velocity and turbulence distributions at nozzle exit. The High Reynolds number nozzle inlet boundary layers of engineering practice are fully turbulent, but acceleration can induce re-laminarisation. Thus, to reproduce nozzle exit conditions accurately, Large Eddy Simulation (LES) for plume prediction must be capable of capturing re-laminarisation and any subsequent boundary layer recovery. Validation is essential to establish a credible LES methodology, but previous studies have suffered from lack of nozzle inlet/exit measurements in the test cases selected. Validation data were here taken from an experiment on a convergent round nozzle with a parallel exit extension to allow boundary layer recovery. LES inlet condition generation applied a Rescaling/Recycling Method $\left(R^{2} M\right)$, whose performance was validated against measurements of $1^{\text {st }}$ and 2 nd moment statistics as well as the turbulence integral length scale. Simulations employed two Sub-Grid-Scale (SGS) models; these produced similar predictions up to the end of the nozzle convergent section, but marked differences appeared for the nozzle exit turbulence field. The Smagorinsky model predicted much lower turbulence levels than measured, whereas the Piomelli \& Geurts model revealed the presence of a small separation region at the convergence/parallel section corner, which led to higher exit turbulence and much better agreement with measured data.
\end{abstract}

\section{KEYWORDS}

Large Eddy Simulation, Nozzle flows, Validation

\section{NOMENCLATURE}

$\begin{array}{llll}A & =\text { Van Driest law constant } & v & =\text { kinematic viscosity } \\ C s & =\text { Smagorinski sgs model constant } & \tau & =\text { unresolved velocity correlation } \\ C_{P G} & =\text { Piomelli and Geurts sgs model constant } & \rho & =\text { density } \\ D & =\text { nozzle exit diameter } & \omega & =\text { vorticity component or magnitude } \\ d x, d y, d z & =\text { grid cell dimensions } & & \\ H_{12} & =\text { shape factor }(=\delta / \theta) & \text { Subscripts } \\ i, j, k, n & =\text { tensor notation indices } & \infty & =\text { free stream property } \\ k & =\text { turbulence kinetic energy } & C L & =\text { centreline property } \\ K & =\text { acceleration parameter } & \delta \text { or e } & =\text { boundary layer edge property } \\ L & =\text { length, integral length scale } & \mathrm{FT} & =\text { flow through time } \\ I & =\text { sgs length scale } & \mathrm{IN} & =\text { inlet property } \\ M a & =\text { Mach number } & \mathrm{PC} & =\text { potential core }\end{array}$

\footnotetext{
${ }^{1}$ Current address: Assistant Professor, Singapore Institute of Technology, Singapore 138683.
} 


$\begin{array}{ll}n & =\text { distance normal to wall } \\ p & =\text { static pressure } \\ R & =\text { gas constant, 2-point correlation } \\ R e & =\text { Reynolds number } \\ S & =\text { strain rate } \\ t & =\text { time } \\ T & =\text { temperature or flow through time } \\ U, V, W & =\text { resolved scale velocity components } \\ u, V, W, & =\text { sub-grid scale velocity components } \\ x, y, z & =\text { spatial co-ordinates } \\ a & =\text { time step weighting factor } \\ \delta & =\text { boundary layer overall thickness } \\ \delta_{\mathrm{ij}} & =\text { Kronecker delta } \\ \Delta & =\text { cube root of local cell volume } \\ \varepsilon & =\text { turbulence dissipation rate } \\ \Theta & =\text { momentum thickness } \\ \kappa & =\text { log-law constant } \\ \mu & =\text { dynamic viscosity }\end{array}$

$$
\begin{array}{ll}
\text { RMS } & =\text { root mean square } \\
T, S & =\text { total or static property } \\
O & =\text { inlet centreline value } \\
i, j, n & =\text { Cartesian tensor indices } \\
S G S & =\text { sub-grid scale }
\end{array}
$$

\section{Superscripts}

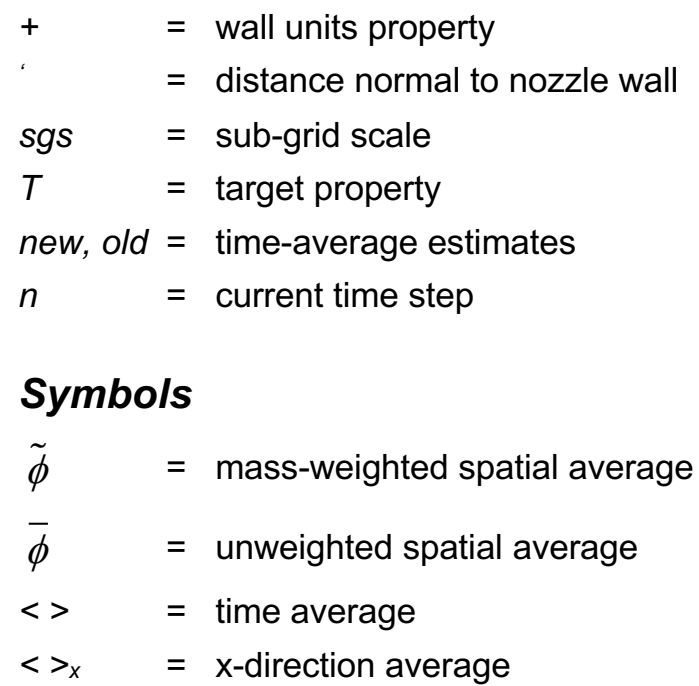

\subsection{INTRODUCTION}

A CFD methodology for propulsion nozzle design, followed by analysis of the resulting jet plume, is a fundamental requirement in civil/military aerospace. The primary motivation is the need to predict hot jet temperature decay and broadband noise sources in order to meet design requirements for Infra-Red signature (military) and reduced jet noise (civil). The jet near-field (approximately first 20 nozzle diameters) presents a more complex technical challenge than the far-field, where jet development obeys self-similarity laws. At exit the nozzle wall boundary layer is converted into firstly a free shear layer and eventually a jet; the early stages of this conversion process are clearly influenced by flow conditions at nozzle exit, implying internal flow development must also be considered. Near-field turbulent structures must be predicted accurately since these control near-field jet aerodynamic and high frequency aeroacoustic characteristics.

A generally applicable CFD methodology for this application has, however, yet to be demonstrated. Reynolds Averaged Navier Stokes (RANS) results have fallen short of the accuracy needed. Improvements have been proposed (Kenzakowski et al. [1]) to industrystandard 2-equation turbulence models but have not demonstrated acceptable accuracy for prediction of the variation of the potential core length $\left(L_{P C}\right)$ with $\mathrm{Ma}_{\lrcorner}$for a general nozzle geometry, a capability essential to design. Georgiadis and DeBonis [2] argued that RANS models cannot reproduce near-field turbulence since their constants were calibrated in far-field self-similar flows. A 'zonal' approach was recommended, simply adjusting model constants to suit round jet near-field data. This is, however, insufficiently robust for all nozzle geometries or relevant flow conditions. There is a growing body of evidence that unsteady flow effects must be included for better and more generally applicable predictions. This has led to Large Eddy Simulation (LES) being increasingly preferred to RANS in detailed aerodynamic/aeroacoustic 
plume studies (DeBonis [3], Karabasov et al. [4]), although at significantly increased computational cost. Wang and McGuirk [5], for example, have demonstrated that, unlike RANS, LES can predict $L_{P C}$ accurately for a supersonic rectangular jet. The crucial role of nozzle exit conditions was particularly emphasised by Bres et al. [6], who confirmed that accurate representation of nozzle exit characteristics is necessary for "significant improvement of flowfield and sound prediction". Given these claims for superior LES performance, prior LES work on jet plumes is reviewed next.

Early LES studies started at nozzle exit with specified (usually guessed) boundary conditions; laminar profiles were often chosen, although unlikely to be appropriate - even if strong flow acceleration has caused relaminarisation, the high overall Reynolds number $(\operatorname{Re})$ of engineering practice (typically $>\mathrm{O}\left(10^{6}\right)$ ) will lead to characteristics resembling a low-Re turbulent boundary layer. Including internal nozzle flow in simulations implies boundary conditions must be specified at nozzle inlet. Chung and Sung [7] showed that inadequate treatment of LES inlet turbulence affects development over a large downstream distance. LES nozzle inflow conditions should ideally correspond to spatially- and temporally-correlated time-series which accurately represent the actual nozzle flow. A comprehensive review of LES inlet condition generation methods has been provided by Tabor and Baba-Ahmadi [8]; the review by Wu [9] describes four approaches: (i) Random Fourier Methods (to create a prescribed turbulence energy spectrum), (ii) Digital Filtering Methods, (iii) Synthetic Eddy Methods, and (iv) Rescaling/Recycling Methods $\left(R^{2} M\right)$ - where a separate ('auxiliary') simulation is performed to generate the required unsteady LES inlet boundary conditions. Morgan et al. [10] compared these and found that the 'recovery' length (the distance for the turbulence to recover from inconsistent or incomplete inlet condition information) is typically shorter for $R^{2} M$, and thus only this approach is reviewed here.

The use of an auxiliary LES solution in $R^{2} M$ ensures the generated inlet velocity is spatially and temporally self-consistent. The disadvantage is the extra computational cost, which must be justified by improved physical realism. Most $R^{2} M$ variants follow Lund et al.'s [11] proposal. Liu and Pletcher [12] and Arya et al. [13] have suggested modifications to address the problem that if rescaling is based on similarity laws but flow at the recycling plane has not reached equilibrium, then incorrect data are recycled, leading to a longer recovery length. Another potentially serious problem is that recycling between spatially separate planes introduces a non-physical spatial correlation (Nikitin [14]). Morgan et al. [10] proposed a method to avoid this by using spanwise scrambling before recycling; this method is successful but limited to flows with spanwise homogeneity.

A good example of use of $R^{2} M$ with relevance to the current work is reported in Piomelli and Yuan [15]. This applies LES to a relaminarising plane wall boundary layer with an imposed accelerating pressure gradient, using $R^{2} M$ to generate an equilibrium turbulent inlet boundary layer of prescribed thickness. It is well known that strong/sustained acceleration causes relaminarisation (Narasimha and Sreenivasan [16]) leading to absence of any equilibrium log-law region. Values of the acceleration parameter $K=\frac{v}{U_{\infty}^{2}} \frac{d U_{\infty}}{d x}$ greater than $3 \times 10^{-6}$ indicate a high probability of re-laminarisation. The Jones and Launder [17] k- $\varepsilon$ model was developed precisely to predict this phenomenon; however, Yang and Tucker [18] noted that, whilst capturing relaminarisation, this model predicts a too rapid return to equilibrium after acceleration is removed. Piomelli and Yuan [15] demonstrated that LES does not suffer from this defect. Good agreement with measurements of Warnack and Fernholtz [19], [20] were obtained as long as mesh spacing allowing a 'wall-resolved simulation' was used. LES results compared favourably with the Reynolds stress development in both re-laminarising and recovery regions. These results for a flat plate with an imposed acceleration are encouraging for the potential of LES in accelerated nozzle flows, although to date this has not been explored. Note, however, that the 
flow Reynolds number in [15] was considerably lower than that of relevance here; achieving a wall-resolved simulation will be challenging at higher Reynolds numbers.

All the above were for developing 2D boundary layers. Pierce and Moin [21] suggested an alternative, more general $R^{2} \mathrm{M}$ technique, falling into the category of 'synthetic volume forcing methods' (Wu [9]). Extra pressure gradient terms were included in the $R^{2} M$ equations to force the unsteady flow to possess user-defined ('target') statistical velocity characteristics, and this approach was applied in [21] to an inlet condition corresponding to a fully-developed swirling annulus flow. Xiao et al. [22] argued that repeated rescaling to force a match to target statistical data achieves the same outcome as extra pressure gradients. Application to $2 \mathrm{D}$ and $3 \mathrm{D}$ boundary layer and free shear flows showed their $R^{2} M$ variant was also able to reproduce correct distributions of important statistical properties that were not part of the input target data (Reynolds shear stress and integral length scale); Li et al. [23] have used the same approach for inlet conditions consisting of a combination of end wall boundary layers and a wake flow.

Previous published LES studies of combined jet nozzle/plume flow have concentrated on the jet noise application. Bogey and Marsden [24] included a nozzle length of only 1 nozzle exit diameter within the LES solution domain. A numerical procedure (akin to trip wires in experiments) injected unsteady fluctuations to 'seed turbulence development in the nozzle wall boundary layer'. Initially random fluctuations superimposed on a laminar boundary layer were chosen; this was later refined to consider 2D azimuthal vortical structures de-correlated in the azimuthal direction. The conclusion drawn was: "it is important in jet simulations to control the initial velocity disturbances, so as to impose nozzle exit conditions as close as possible to experimental conditions when they are known". A similar approach had been earlier applied by Pokora et al. [25] and improved prediction of 2-point correlations in the initial jet shear layer compared to measurements. This approach is superior to simulations which simply prescribe the nozzle exit conditions, but it is not clear if it could handle re-laminarised and recovering boundary layers, or match measurements of exit flow Reynolds shear stress/turbulence length scale if these were available.

Uzun et al. [26], Fosso-Pouange et al. [27] and Bres et al. [6], [28] have all included nozzle geometry in their simulations. Various mesh systems, numerical discretisations, inlet condition treatments and sub-grid-scale (SGS) models have been adopted (e.g. no SGS model in [26], the Wale model in [27] and the Vreman model in [6] and [28]). In [26] only a short length of cylindrical pipe was included for the nozzle although he experimental geometry comprised a longer convergent pipe; the nozzle Re was also lowered by a factor of $\sim 15$ compared to the experiment to ease numerical resolution; this may be acceptable for Re-independent far-field jet flow, but is not sensible for internal nozzle flow. In terms of the nozzle inlet condition treatment, [26] used guessed profiles; various boundary layer thicknesses were tried, and the near-field solution was (inevitably) sensitive to this. [27] adopted a Bogey and Marsden [24] approach, but this failed to produce a turbulent nozzle exit boundary layer, probably because the chosen amplitude of disturbance was too small to survive the nozzle acceleration.

The LES approach adopted in [6] and [28] represents the most comprehensive proposal to date for nozzle/jet plume simulations. This work concluded that: (i) a digital filtering approach to generate synthetic turbulence in nozzle inlet conditions, and (ii) a hybrid 1D RANS/LES method for near-wall cells (labelled 'wall modelling') were essential elements. These reduce the need for a very fine near-wall LES mesh and avoid the (unphysical) laminar nozzle exit boundary layer which occurs if synthetic inlet turbulence/wall modelling are excluded. Inclusion of these enabled improved agreement with measured near-field flow development (better prediction of $L_{C}$ ) and more accurate capture of the high frequency region of noise spectra. However, this approach still has some deficiencies. In [6] the wall model failed to reproduce the near-wall Reynolds stress anisotropy in a zero pressure gradient boundary layer. In [28] the test 'nozzle' included in the simulation comprised only a straight cylindrical pipe, neglecting the acceleration effects occurring in real nozzles. The synthetic inlet turbulence/wall model were 
thus (effectively) used to ensure velocity and axial normal stress profiles measured 0.04D downstream of exit (produced by a roughness strip included in the experiment just upstream of exit) were matched as closely as possible. The peak value of the Reynolds stress was still overpredicted by $25 \%$ and agreement with the rest of the stress profile was only achieved by adjusting the amplitude of the inlet synthetic turbulence. Finally, since no measurements of either mean velocity or turbulence were available at the start of the 10D long straight 'nozzle', validation of the predicted internal 'nozzle' flow development from known starting conditions was not considered.

In summary, previous LES work displays gaps/deficiencies in several areas and no methodology has clearly demonstrated a validated capability to predict the nozzle exit flow characteristics accurately. The present contribution describes work to address this issue. Whilst the ultimate objective is a methodology for combined nozzle/jet plume flow, the importance of correct nozzle exit conditions (Bres et al. [6]) means emphasis is here concentrated on validation of isolated accelerated nozzle flow. An experimental study providing suitable validation data was recently published by Trumper et al. [29]. Data were reported at Reynolds and Mach numbers representative of full-scale conditions and profile information provided at both nozzle inlet and exit; further justification that this experiment is an appropriate validation test case is provided below. The data are used to validate an LES methodology based on: (i) the use of the Xiao et al. [22] $R^{2} \mathrm{M}$ variant for nozzle inlet conditions, (ii) an adequately-resolved near wall mesh, and (iii) a flexible but simple eddy viscosity SGS model to capture the flow physics of the internal nozzle flow.

\subsection{JUSTIFICATION OF CHOSEN VALIDATION TEST CASE}

Trumper et al. [29] report experiments for a range of high subsonic and underexpanded NPRs from an axisymmetric convergent nozzle including capture of the nozzle inlet, exit, and near-field jet development. All data were taken with jet total temperature equal to ambient static temperature (typically 293K). Full details of facility, instrumentation and measurements are provided in [29]; emphasis is here placed on the quality and suitability of these data as a validation test case for current purposes. Details are provided of: (i) nozzle geometry, (ii) range of operating parameters investigated, (iii) sample data at nozzle inlet/exit and (iv) the flow condition chosen for study.

The datum nozzle geometry (labelled LU60) had an internal convergence half angle of $11^{0}$ and exit diameter $D=60 \mathrm{~mm}$; a short upstream inlet section (length $51.0 \mathrm{~mm}, \mathrm{OD}=86 \mathrm{~mm}$, ID = $75 \mathrm{~mm}$ ) was provided for attachment onto a high pressure air supply pipe. A second nozzle (LU60P, Fig.1) added a 31.8mm parallel exit extension to the datum geometry (exit lip thickness $1 \mathrm{~mm}$ ). This removed vena contracta effects and allowed boundary layer recovery from internal nozzle acceleration.

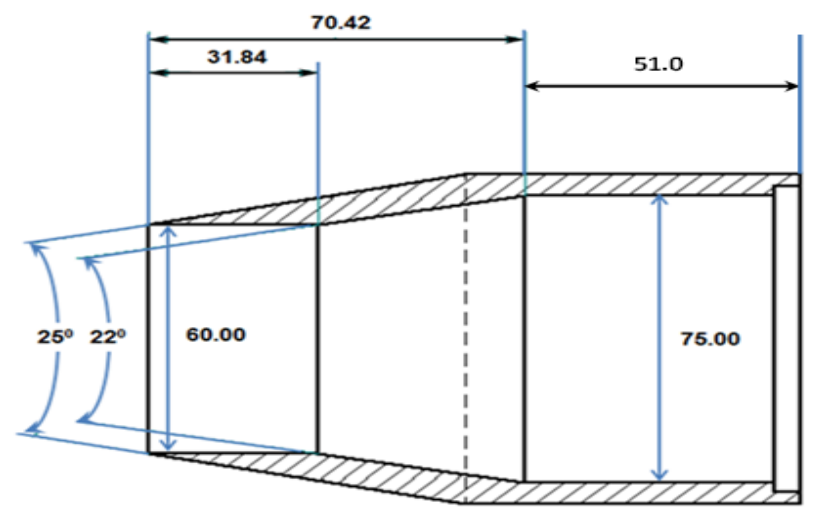

Fig. 1 Chosen test problem - axisymmetric convergent nozzle LU60P, dimensions in mm. 
The LU60P geometry was chosen for use in the current work since it possessed both accelerating and recovery regions and was thus a more challenging test case. The nozzle was operated at NPRs from 1.3 to 2.4 (subsonic to moderately underexpanded), with jet exit Mach numbers $\mathrm{Ma}_{\lrcorner}=0.56$ to $\mathrm{Ma}_{\mathrm{J}} \sim 1.2$ (fully expanded), and corresponding inlet Mach numbers $\mathrm{Ma}_{\mathrm{IN}}$ $=0.35-0.46$. Jet Reynolds numbers were representative of engineering applications $\left(010^{6}\right)$. The acceleration parameter $K$ was $\sim 1.5 \times 10^{-6}$ for most of the nozzle but rose to $\sim 5 \times 10^{-6}$ for the final $15 \%$ of convergence. Pneumatic probe measurements were made $30 \mathrm{~mm}$ (0.5D) upstream of the start of convergence, which is thus treated here as the nozzle inlet plane; LDA measurements were made at nozzle exit. [29] contains detailed descriptions and definitions of all measured quantities and the same definitions have been used in the LES when comparing with measurements.

Due to a long upstream air supply duct in the test facility, a thick nozzle inlet boundary layer of overall thickness $\sim 20 \mathrm{~mm}$ was observed at all NPRs tested ( $53 \%$ of inlet radius). Note, this is substantially thicker than would occur at typical engine nozzle operating conditions. A better approach might have been first to carry out CFD predictions for a solution domain which was representative of the engine duct geometry feeding the nozzle, including at least some of the upstream turbomachinery. This would provide useful information about the characteristics of the flow entering the nozzle. A laboratory facility upstream supply duct could then be designed to match the engine-representative approach flow/boundary layer thickness This is clearly a worthwhile topic for future work, but for the present study the use of the simple inlet boundary layer profile measured in [29] was considered acceptable. The objective of the current work was to demonstrate a working LES methodology for predicting accelerating nozzle flows with known nozzle inlet conditions, experiencing an acceleration typical of that found in propulsion nozzles and able to successfully predict the nozzle exit characteristics; the data of [29] were considered appropriate for this. Inlet conditions are unlikely to have been measured in many engineering applications, so the CFD approach outlined above would then undoubtedly have to be applied.

To illustrate the fully turbulent nature of the inflow profile, Fig. 2(a) presents this in wall law co-ordinates $\left(\mathrm{u}^{+}\right.$vs $\left.\mathrm{y}^{+}\right)$, and Fig. 2(b) shows measured shape factor $\left(H_{12}\right)$. The former displays close agreement with the standard log-law and the latter shows a value corresponding to a fully

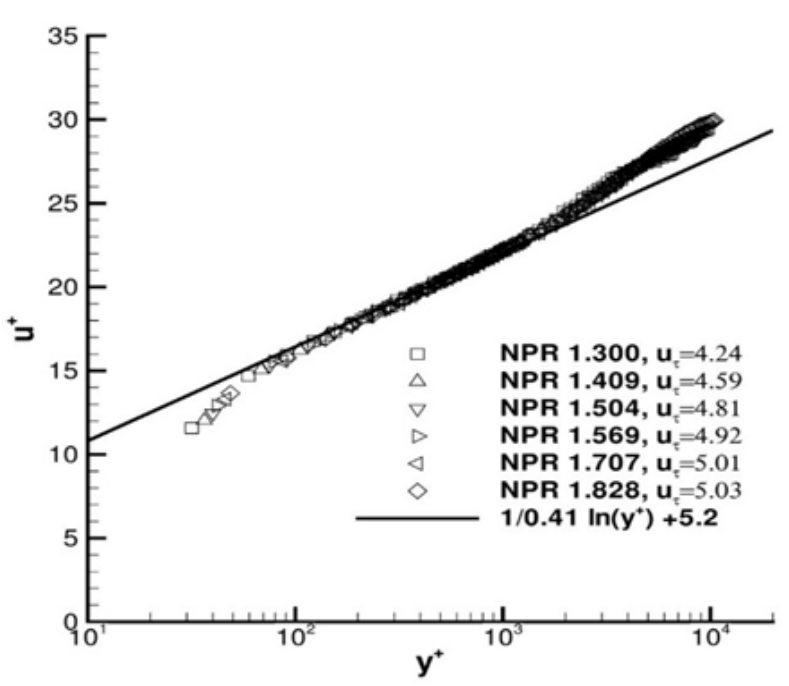

Fig. 2a Nozzle inlet mean velocity profile (Trumper et al. [29])

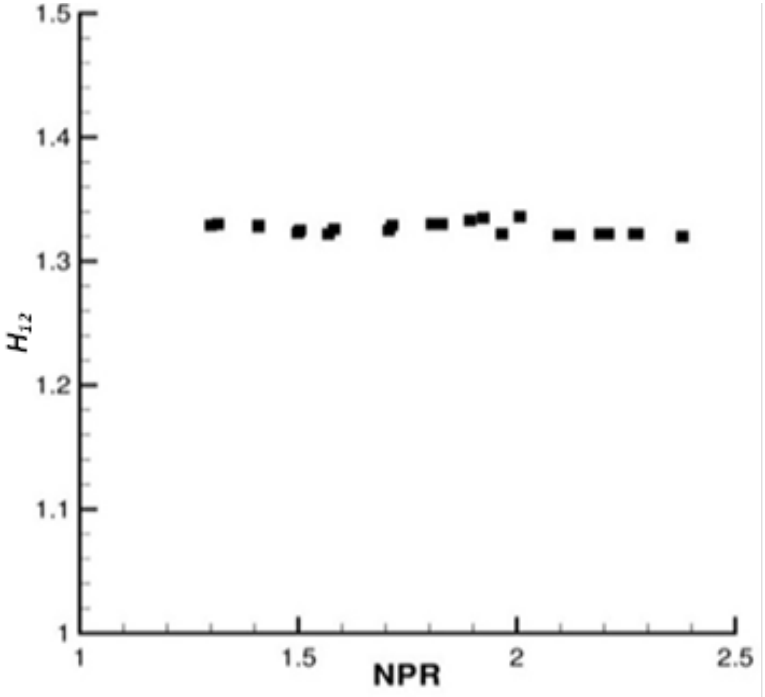

Fig. 2b Nozzle inlet shape factor $\mathrm{H}_{12}$ (Trumper et al. [29]) 
turbulent profile $\left(\mathrm{H}_{12}=1.33+/-0.01\right)$ for all NPRs. This demonstrates clearly that the nozzle inlet conditions corresponded closely to an equilibrium fully turbulent boundary layer. Note that the data in Fig. 2 are for the clean LU60 nozzle geometry; it was stated in [29] that "it was not expected that the addition of the exit extension would influence inlet profiles" and this was confirmed in the simulations shown below. Although turbulence statistics were not measured, in [29], the mean flow characteristics observed imply that the plentiful data available in the literature for Reynolds stress distributions in zero pressure gradient boundary layers may reasonably be added to the mean velocity data of Fig. 2 to provide a complete nozzle inlet specification, as required to make this a suitable validation data set; how this was achieved in the current work is described below.

At nozzle exit the measured data revealed that the internal acceleration had led to a dramatic reduction in boundary layer thickness and a laminar-like profile shape for the LU60 design. For the LU60P geometry, the short exit extension allowed recovery to a turbulent state and increased thickness considerably (Table 1). Measured inlet values of $\delta^{*}$ and $\theta$ were $2.04 \mathrm{~mm}$ and

Table 1 LU60/LU60P boundary layer parameters (NPR $=1.5$, Trumper et al. [29])

\begin{tabular}{|c|c|c|c|c|}
\hline & $\delta^{*}(\mathbf{m m})$ & $\theta(\mathbf{m m})$ & $\boldsymbol{R e}_{\boldsymbol{\theta}}$ & $\boldsymbol{H}_{\mathbf{1 2}}$ \\
\hline LU60 & 0.065 & 0.0248 & 442 & 2.00 \\
\hline LU60P & 0.44 & 0.362 & 7494 & 1.22 \\
\hline
\end{tabular}

$1.45 \mathrm{~mm}$ (at NPR $=1.50$ ) with $\mathrm{Re}_{\theta}=18,800$ and $\mathrm{H}_{12}=1.33$ for both nozzles. Table 1 shows LU60 exit thickness parameters were drastically reduced, with exit $\mathrm{H}_{12}=2.0$ close to that of a fully laminar boundary layer. The very low exit $\mathrm{Re}_{\theta}$ is in the range described by Fernholz and Finley [30] as constituting a "low Reynolds number transitional boundary layer". The acceleration within the nozzle has clearly created a re-laminarised boundary layer. Data for the LU60P geometry shows the exit boundary layer has recovered substantially with $\theta \sim 15$ times larger than for the convergence-only nozzle; $\mathrm{H}_{12}$ also now displays a turbulent value -1.22 . It was also reported in [29] that, when plotted in semi-logarithmic Clauser form, the exit profile for the LU60P nozzle displayed clear evidence of recovery towards a fully turbulent equilibrium shape. However, in the near-wall region of the profile $\left(\mathrm{y}^{+} \sim 10^{3}\right)$ measurements deviated considerably further from the equilibrium turbulent line than at points further away from the wall. It was suggested in [29], on the basis of arguments outlined in [31], that this was considered to be evidence of local adverse pressure gradient effects and was most likely associated with "flow turning associated with the internal corner between the convergent nozzle wall and the parallel extension wall". It is likely that such localised effects will be challenging to capture in CFD predictions. This is particularly so since the strength of these adverse pressure gradient effects will be strongly dependent on the precise geometry at the junction between convergent and parallel extension walls, which is not detailed in [29].

Finally, LDA measured nozzle exit mean axial velocity and rms turbulence profiles are presented in Fig. 3 for two NPRs (note that profiles non-dimensionalised using boundary layer edge velocity $\bigcup_{\delta}$ and $\delta^{*}$ ). Fig. 3a shows that the LU60P exit flow possessed a fuller shape with a steeper near wall gradient. Fig. $3 b$ for the axial normal stress indicates boundary layer recovery in the exit extension has led to a decrease in peak value and a shift of the peak to very close to the wall.

Examination of Figs 2 and 3 indicates clearly the suitability of the experimental data as a validation data set for the present purpose. Nozzle inlet measurements remove the uncertainty in previous studies and exit measurements for both mean and turbulence properties provide clear evidence of the re-laminarisation and recovery effects occurring in the LU60P geometry. The data are thus considered a suitable validation test case for assessment of the predictive 
capability of LES for accelerated nozzle flows. The operating point chosen for study was NPR = $1.5\left(\mathrm{Ma}_{\mathrm{IN}}=0.41, \mathrm{Ma}_{\mathrm{J}}=0.71\right)$ with a total temperature $\tilde{T}_{t}=293 \mathrm{~K}$ and a nozzle inlet centreline velocity $U_{0}=139 \mathrm{~m} / \mathrm{s}$.

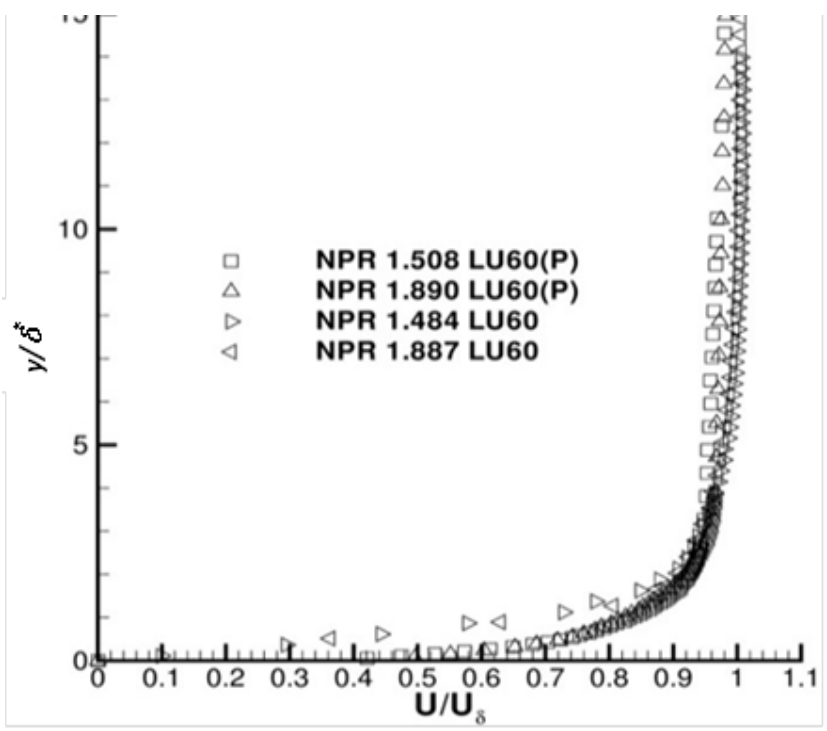

Fig. 3a LU60/LU60P nozzle exit mean axial velocity profiles, Trumper et al. [29]

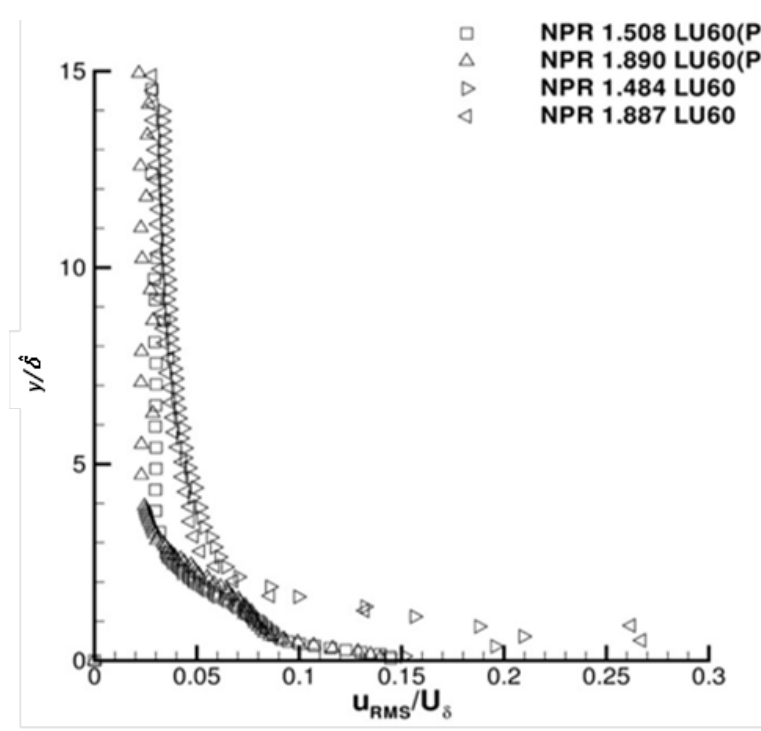

Fig. 3b LU60/LU60P nozzle exit urms profiles, Trumper et al. [29]

\subsection{COMPUTATIONAL METHODOLOGY}

\subsection{LES Formulation}

Mass-weighted low-pass spatial filtering is applied to obtain the Cartesian tensor version of the compressible LES equations. The present flow is subsonic and adiabatic with jet total and ambient static temperatures equal. Thus, the same treatment of the energy equation has been adopted as in the LES simulations of Karabasov et al [4] (Ma $=0.9)$ and Wang and McGuirk [5] (supersonic jet), namely that the steady state solution of constant total enthalpy (or temperature) is a good approximation even in an unsteady flow. This produced good agreement with measurements of both mean and turbulence properties in these earlier works and is assumed here for the chosen NPR $=1.5$ test case $\left(\mathrm{Ma}_{J}=0.71\right)$. The static temperature $\left(\tilde{T}_{s}\right)$ is calculated from the specified inlet total temperature $\left(\tilde{T}_{t}\right)$ and predicted velocity field; the density is evaluated from the equation of state for an ideal gas. The governing equations solved were:

$$
\begin{gathered}
\frac{\partial \bar{\rho}}{\partial t}+\frac{\partial}{\partial x_{j}}\left(\bar{\rho} \tilde{u}_{i}\right)=0 \\
\frac{\partial}{\partial t}\left(\bar{\rho} \tilde{u}_{i}\right)+\frac{\partial}{\partial x_{j}}\left(\bar{\rho} \tilde{u}_{j} \tilde{u}_{i}\right)=-\frac{\partial \bar{p}}{\partial x_{i}}+\frac{\partial}{\partial x_{j}}\left[\mu\left(\frac{\partial \tilde{u}_{i}}{\partial x_{j}}+\frac{\partial \tilde{u}_{j}}{\partial x_{i}}\right)-\frac{1}{3} \frac{\partial \tilde{u}_{k}}{\partial x_{k}} \delta_{i j}\right]-\frac{\partial}{\partial x_{j}}\left(\overline{\rho u_{i} u_{j}}-\bar{\rho} \tilde{u}_{i} \tilde{u}_{j}\right) \\
\bar{p}=\bar{\rho} R \tilde{T}_{s}
\end{gathered}
$$


where $\left(\overline{\rho u_{i} u_{j}}-\bar{\rho} \tilde{u}_{i} \tilde{u}_{j}\right)$ is the residual (SGS) stress tensor $\tau_{i j}^{s g s}$. To close the momentum equations two SGS models were used; the first is the standard Smagorinsky [32] model:

$$
\begin{gathered}
\tau_{i j}^{s g s}=\left(\overline{\rho u_{i} u} \bar{\rho}_{j}-\tilde{u}_{i} \tilde{u}_{j}\right)=-2 \mu_{s g s} \tilde{S}_{i j}+\frac{2}{3} \bar{\rho} \tilde{\mathrm{k}}_{s g s} \delta_{i j} \\
\tilde{\mathrm{S}}_{i j}=\frac{1}{2}\left(\frac{\partial \tilde{u}_{i}}{\partial x_{j}}+\frac{\partial \tilde{u}_{j}}{\partial x_{i}}\right) \quad \mu_{s g s}=\bar{\rho} l_{s g s}^{2} S \quad S=\left(2 \tilde{S}_{i j} \tilde{S}_{i j}\right)^{\frac{1}{2}} \\
l_{s g s}=C_{S} \Delta \quad \Delta=(\delta \mathrm{x} \delta \mathrm{y} \delta \mathrm{z})^{1 / 3} \quad \text { and near walls: } \quad l_{s g s}=C_{S} \Delta\left(1-e^{n^{+}} / A^{+}\right)
\end{gathered}
$$

where $I_{\text {sgs }}$ is the SGS length scale and $\Delta$ is a mesh-based length scale (cube root of local cell volume). In the near wall region van Driest [33] damping is used: $\mathrm{n}^{+}$is wall normal distance and $A^{+}=26$. Literature reveals a wide range $(0.09-0.18)$ can be found for the Smagorinsky constant $C_{S}$ with a tendency for the upper end; in the present work $C_{s}$ was set to 0.15 as in earlier LES studies [4] and [5].

The second SGS model follows Piomelli and Geurts [34]; this retains the SGS viscosity definition but calculates $I_{\text {sgs }}$ differently. The motivation is to avoid the link between $I_{\text {sgs }}$ and mesh size, which has several undesirable features (Piomelli et al. [35]). In the Piomelli and Geurts [34] model $I_{\text {sgs }}$ is set proportional to a fraction of the (estimated) integral length scale $L$; dimensional analysis relates this to the resolved scale turbulence energy and vorticity magnitude:

$$
L \propto \frac{k^{1 / 2}}{\omega} \quad \text { where: } \quad k=\frac{1}{2}\left\langle\left(\tilde{\mathrm{u}}_{i}-<\tilde{\mathrm{u}}_{i}>\right)\left(\tilde{\mathrm{u}}_{i}-<\tilde{\mathrm{u}}_{i}>\right)\right\rangle \quad \omega=\left\langle\tilde{\omega}_{i} \tilde{\omega}_{i}\right\rangle^{1 / 2}
$$

Both $\mathrm{k}$ and $\omega$ are statistically averaged since $L$ is a statistically averaged quantity. Resolved rather than fluctuating vorticity is used [34] to ensure that in a laminar boundary layer the turbulence length scale is zero. Piomelli et al. [35] have noted that the calculation of $L$ can be improved by including SGS quantities and proposed a modification along these lines. This version has, however, so far only been tested in homogeneous isotropic turbulence and fully developed channel flow, and the earlier [34] model was adopted in the current work. SGS length scale and viscosity are calculated from:

$$
l_{s g s} \propto L \propto \frac{k^{\frac{1}{2}}}{\omega}=C_{P G} \frac{k^{\frac{1}{2}}}{\omega} \quad \quad \mu_{s g s}=\bar{\rho} C_{P G}^{2} \frac{k}{\omega^{2}} S
$$

$\mathrm{C}_{P G}$ is a model coefficient, determined by Piomelli and Geurts [34] in a parametric study of a fully-developed channel flow to minimise error in the skin friction coefficient. The minimum was found for $\mathrm{C}_{P G}=0.12$, although, in the flow considered, the error proved insensitive to variations over the range of $0.05-0.15$. The model has not previously been applied to a convergent duct flow and, given the wide range of optimum value for $\mathrm{C}_{P G}$, further consideration in choosing a value for $C_{P G}$ was deemed appropriate. Piomelli et al. [35] suggested that: "the optimum value of the coefficient may be chosen to minimise the total simulation error in prediction of a set of selected quantities known from experiments". Since the integral length scale $L$ plays a dominant role in the model, the value of $C_{P G}$ is here determined by minimising the error in prediction of $L$ in a zero pressure gradient boundary layer (see Section 4.2). 


\subsection{CFD Code Details}

The CFD code used adopts a hexahedral, cell-centred, multi-block, structured mesh and a pressure-based approach. Originally written for RANS calculations of compressible flows it was converted to LES for prediction of multiple impinging jets in Page et al. [36]. The same spatial and temporal discretisation schemes were adopted as in the LES studies of Karabasov et al. [4] and Wang and McGuirk [5]. $2^{\text {nd }}$ order spatial discretisation was employed, and temporal advancement made using a low-storage Runge-Kutta $4^{\text {th }}$-order (5-stage) scheme; in all simulations the maximum CFL was always less than 0.25. References [4] and [5] provide evidence of acceptable numerical robustness and dispersion/dissipation characteristics.

\section{3 $\mathrm{R}^{2} \mathrm{M}$ Approach for LES Inlet Condition Generation}

The Xiao et al. [22] $\mathrm{R}^{2} \mathrm{M}$ technique was applied in the present work. This attaches an 'Inlet Condition' (IC) domain to a 'Main Simulation' (MS) domain (i.e. the nozzle of Fig.1, see Fig.4). Rescaling/recycling are used to match specified target statistics throughout the IC domain, within which statistical properties are assumed streamwise homogeneous, effectively implying that the MS domain inflow conditions are changing only slowly axially. The IC domain length is chosen purely to ensure streamwise turbulent structures (axial 2-point correlations) are not constrained by IC domain end boundary conditions (after statistical convergence of the simulation this has been retrospectively checked (see Section 4.2). Fig.4 shows reference plane labelling: IC domain inlet/outlet: $A(x / D=0), C(x / D=2.5)$ (also MS domain inlet); re-cycling plane $B(x / D=2.08)$, and $D, E$, and $F$ at convergence start/end $(x / D=3.0, x / D=3.64)$, and nozzle exit $(x / D=4.17)$.

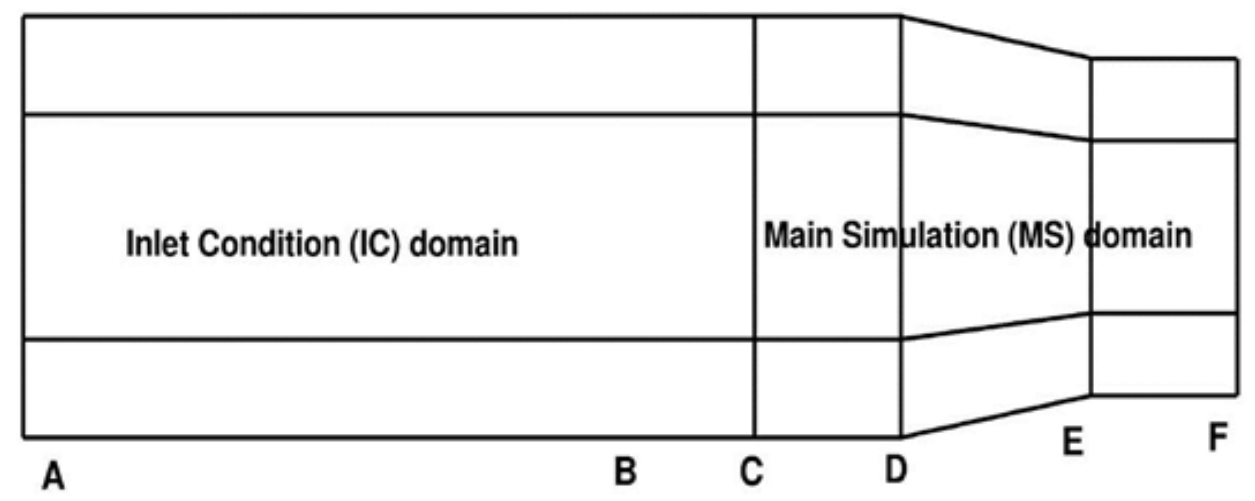

Fig. 4: Inlet Condition and Main Simulation (nozzle) domains

The method generates spatially- and temporally-correlated time-series, which are consistent with specified ('target') statistical properties (mean velocity $U_{i}^{T}$ and 3 turbulence normal stresses (rms values $u_{r m s, i}^{T}$ ) in Cartesian components) chosen to match measurements. The nozzle inlet duct is axisymmetric and the mean velocity in the measurements of [29] is thus available on a single radial line in cylindrical polar co-ordinates $\left(U_{x}=f(r), U_{r}=U_{\theta}=0\right)$; radial profiles for the associated turbulence rms values (which were not measured in [29]), were created by rescaling boundary layer DNS data from Spalart [37] as described next (NB, this therefore constitutes an approximation in the inlet data.) 
A low-Re k- $\varepsilon$ RANS prediction of flow in a long pipe of the same diameter as the inlet duct was first carried out starting from a flat velocity profile corresponding to the experimentally measured mass flow for NPR $=1.5$. A downstream location in this pipe was then selected where the predicted mean axial velocity best matched the measured target data profile. The Spalart data were then rescaled to create axisymmetric target turbulence rms data using eqn. (9) below (only radial component shown for illustration).

$$
\left[\sqrt{\overline{u_{r}^{2}}}\right]_{t a r g e t}(r)=\left[\sqrt{\overline{u_{r}^{2}}}\right]_{\text {SPALART }}(r)\left[\frac{k_{\text {RANS }}^{1 / 2}(r)}{k_{\text {SPALART }}^{1 / 2}(r)}\right]
$$

Finally, since the LES code solves for Cartesian components, the statistical values for the $1^{\text {st }}$ and $2^{\text {nd }}$ moments in cylindrical polar form were transformed to Cartesian components using standard relations.

The $R^{2} M$ technique was then implemented as follows:

(i) Every $\mathrm{n}^{\text {th }}$ time step new estimates of statistical moments of velocity were evaluated:

$$
\begin{gathered}
\left\langle\tilde{u}_{i}\right\rangle^{\text {new }}(y, z)=\alpha\left\langle\tilde{u}_{i}^{n}\left(x, y, z, t_{n}\right)\right\rangle_{x}+(1-\alpha)\left\langle\tilde{u}_{i}\right\rangle^{\text {old }}(y, z) \\
u_{r m s, i}^{\text {new }}(y, z)=\left(\alpha\left\langle\left[\tilde{u}_{i}^{n}\left(x, y, z, t_{n}\right)-\left\langle\tilde{u}_{i}\right\rangle^{n}(y, z)\right]^{2}\right\rangle_{x}+(1-\alpha)\left[u_{r m s, i}^{\text {old }}(y, z)\right]^{2}\right)^{1 / 2}
\end{gathered}
$$

(ii) The instantaneous velocity at all mesh points within the IC domain was then rescaled using (10) and (11) and the target statistical data:

$$
\tilde{u}_{i}^{n}\left(x, y, z, t_{n}\right)=\frac{u_{r m s, i}^{T}(y, z)}{u_{r m s, i}^{\text {new }}(y, z)}\left[\tilde{u}_{i}^{n}\left(x, y, z, t_{n}\right)-\left\langle\tilde{u}_{i}\right\rangle^{\text {new }}(y, z)\right]+U_{i}^{T}(y, z)
$$

(iii) Finally, the velocity field obtained from (12) was recycled from plane B to A and the next time step begun.

When the IC simulation has reached a statistically stationary state, the transfer of IC domain data to the MS domain inlet (plane $C$ ) ensures a time-varying inlet condition for the main simulation with the desired statistics and self-consistent spatial/temporal correlations.

\subsection{Mesh Design}

Fig. 5 presents the multi-block mesh structure adopted (alternate lines only); the block topology of central $\mathrm{H}$-grid and outer O-grids (4 blocks) is optimal for axisymmetric geometry and

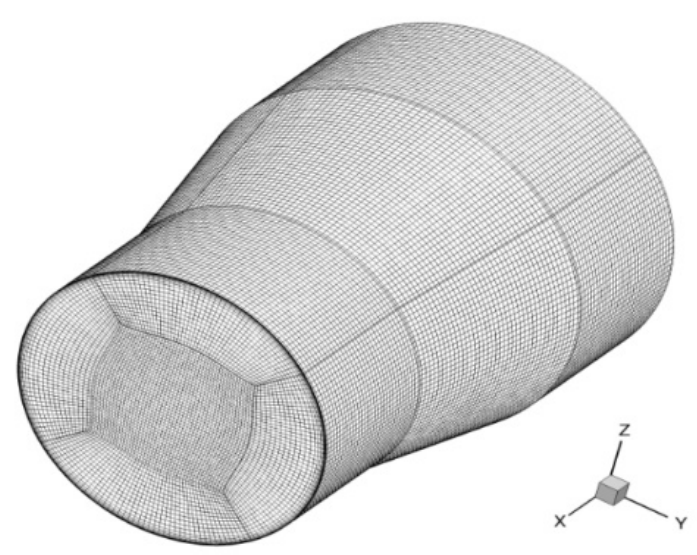

Fig. 5a Nozzle geometry/mesh

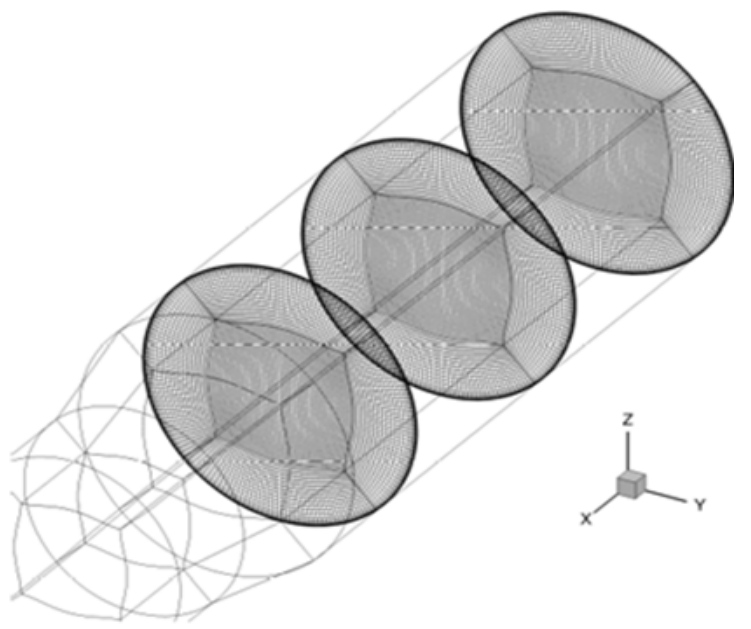

Fig. 5b IC domain mesh 
avoidance of centreline stiffness. LES mesh design followed guidelines using a-priori RANS solutions (low Re Launder-Sharma model used here) as in the approach proposed by Gant [38] and Dianat et al. [39]. The first guideline was used to select a mesh density appropriate for resolution of turbulence structures in regions not too close to a wall. Pope [40] has proposed that for a 'well-resolved simulation' in such regions the mesh should capture greater than $80 \%$ of fluctuating energy, a criterion also used by Celik et al. [41] and shown in Dianat et al. [39] to imply that $\mathrm{L} / \Delta$ should be $>12\left(\mathrm{~L}=\mathrm{k}^{3 / 2} / \varepsilon\right.$ and $\Delta=$ mesh size $)$.

The second guideline was used to design the near-wall mesh design. Choi and Moin [42] have reported that typical near-wall cell sizes for 'wall-resolved simulations' have varied widely in published LES studies: $\Delta \mathrm{x}^{+}=50-130, \Delta \mathrm{y}^{+}<1$ with 10-20 nodes in the boundary layer, and $\Delta \mathrm{z}^{+}$ $=15-30$ (for streamwise, wall normal, wall parallel directions).

Preliminary RANS calculations on various meshes were carried out to produce a final mesh that satisfied the $\mathrm{L} / \Delta$ criterion at mesh nodes greater than $10 \% \mathrm{D}$ from the nozzle wall, and had a near-wall mesh with $\Delta \mathrm{x}^{+} \sim 200, \Delta \mathrm{z}^{+} \sim 30$, and a first node next to the wall at $\Delta \mathrm{y}+\sim 0.3$. With 65 nodes across the boundary layer at nozzle inlet, 24 at end of convergence, and 55 at nozzle exit the resolution of the boundary layer is significantly finer than that used by Bres et al. [28] (only 10-20 cells inside the boundary layer). The streamwise mesh is larger than recommended, but examination of results at nozzle inlet (see Section 4.2) showed good agreement with mean velocity, turbulence normal stress and integral length scale measurements from equilibrium boundary layer data. Further, Warnack and Fernholz [20] observed streamwise length scales grow significantly in accelerated boundary layers (by a factor of 4 in the experiments of [20] which had a slightly lower $K_{\max }$ than in the Trumper et al [29] data), and a moderate increase in $\Delta \mathrm{x}^{+}$was thus considered acceptable. The final nozzle mesh had $100 \times 90 \times 90$ nodes ( $\mathrm{x}, \mathrm{y}, \mathrm{z}$ directions) in the H-grid and 100x60x90 nodes in each of the 4 outer blocks, a total of 3 million cells. The IC domain length was chosen to be 5 inlet boundary layer thicknesses and his mesh increased total grid size to 4.6 million cells. Overall this is a similar mesh density as used by Bres et al. [6], whose nozzle geometry was 4 times as long as that considered here.

At nozzle inlet a spatially and temporally varying total pressure was applied; this was evaluated at each time step by extrapolating the static pressure upstream from cells just inside the inlet plane and converting this to $\mathrm{P}_{\mathrm{T}}$ using an instantaneous Mach No. calculated from a velocity extracted from the $R^{2} \mathrm{M}$ procedure. Simulations were run for $10 \mathrm{~T}_{\mathrm{FT}}$ (calculated using $\mathrm{U}_{0}$ ) before sampling for statistical properties (every 50 time steps) was carried out for a further $10 \mathrm{~T}_{\mathrm{FT}}$ (a $20 \%$ increase in averaging time showed no influence on statistics).

\subsection{LES VALIDATION STUDY}

\subsection{Optimisation of Piomelli and Geuerts SGS Model Constant}

The Piomelli and Geurts SGS coefficient $C_{P G}$ was optimised for boundary layer flow using simulations carried out in the IC domain alone. Target data comprised the measured inlet mean velocity and turbulence normal stress profiles as detailed in Section 3.3. Simulations were conducted with several $C_{P G}$ values. When a statistically stationary state had been reached the spatial 2-point axial correlation with varying axial separation $R_{11}$ was evaluated on a plane within the IC domain (at $x / D=0.415)$. The axial integral scale $L_{11}$ was then calculated from $R_{11}$ :

$$
\begin{aligned}
R_{11}(x, y, z, d x) & =\frac{\langle u(x, y, z, t) u(x+d x, y, z, t)\rangle}{\sqrt{\left\langle u^{2}(x, y, z, t\rangle\right.} \sqrt{\left\langle u^{2}(x+d x, y, z, t\rangle\right.}} \\
L_{11}(x, y, z) & =\int_{0}^{\infty} R_{11}\left(x, y, z, d x^{\prime}\right) d x^{\prime}
\end{aligned}
$$

Figure 6 shows the correlation function for two points in the boundary layer one near the wall $\left(y^{+} \sim 20\right)$ and one near the boundary layer outer edge $\left(y^{\prime} / \delta=0.95\right)$. 


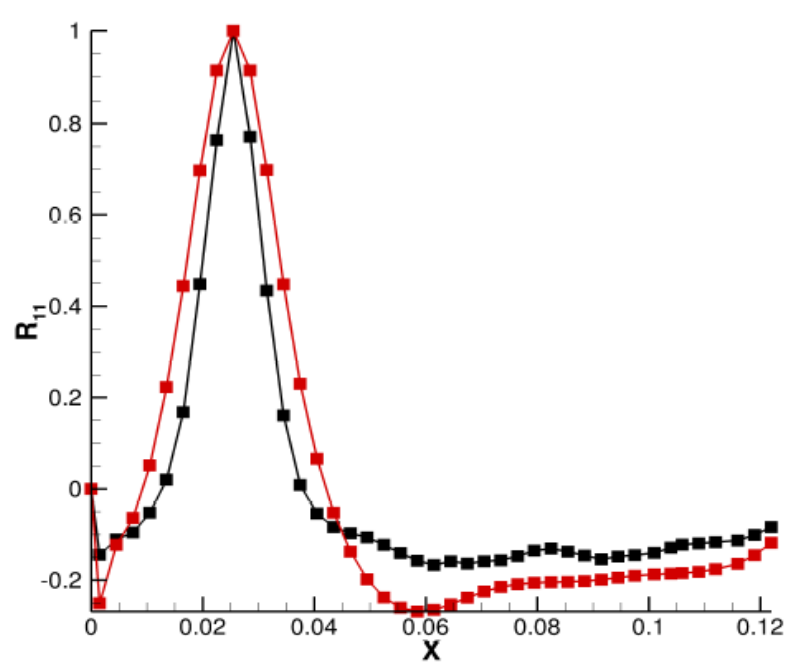

Fig. 6 2-point spatial correlation near wall (black), near outer edge (red) ( $x$ measured in $\mathrm{m}$ from IC domain inlet)

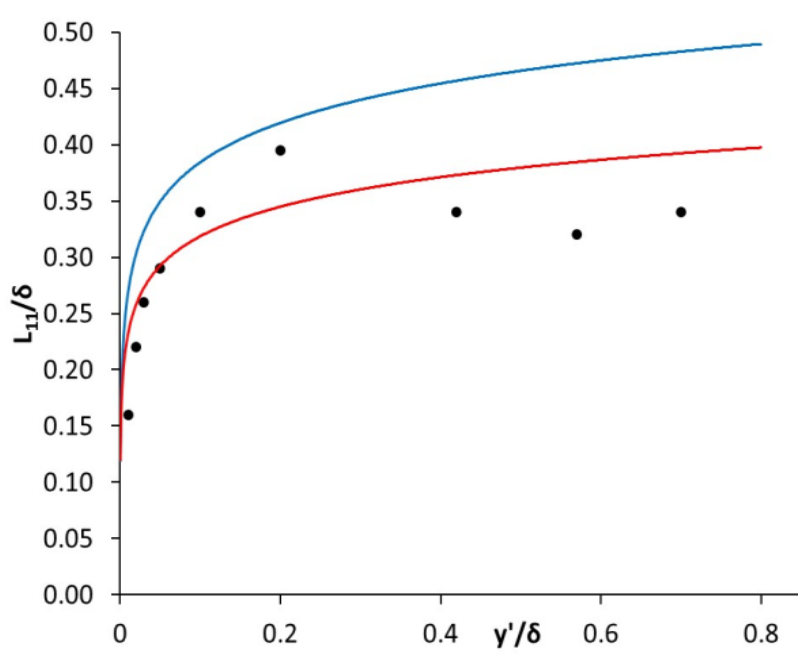

Fig. 7 Axial integral length scale Symbols - Antonia and Luxton [43] data Lines - LES $\left(C_{P G}\right.$ : blue $=0.12$, red $\left.=0.01\right)$

The correlation functions have their first zero crossing well within the IC domain length (this was used as the integration upper limit in evaluating length scales). Thus, the IC domain size seems acceptable for calculating the integral scale, although to capture the correlation shape at large separations as the correlation asymptotes to zero a longer domain would be preferable. Figure 7 shows the predicted variation of $L_{11}$ across the boundary layer for two values of $C_{P G}$ ( 5 values in all were explored between 0.005 and 0.12 spanning the low error range observed in Piomelli and Guerts [33], although only two cases are shown here for clarity). Figure7 indicates that $\mathrm{C}_{\mathrm{PG}}$ $=0.01$ gives best agreement for the integral length scale when judged against the boundary layer data of Antonia and Luxton [43]. In terms of near wall gradient and the value at the boundary layer outer edge $C_{P G}=0.01$ clearly shows best performance. Note the value of $L_{11}$ at the layer edge is quite sensitive to $\mathrm{C}_{\mathrm{PG}}$, unlike the skin friction parameter in the channel flow case. Based on these observations, all simulations below were conducted with $\mathrm{C}_{\mathrm{PG}}=0.01$.

\subsection{Assessment of $R^{2} M$ Method Performance against Measured Data}

Figure 8 shows predicted mean axial velocity contours within the IC domain and part of the MS domain on plane $z / D=0$ (left) and plane $C$ (right). Contours on the first plane illustrate that streamwise homogeneous flow is produced within the whole IC domain, with constant boundary
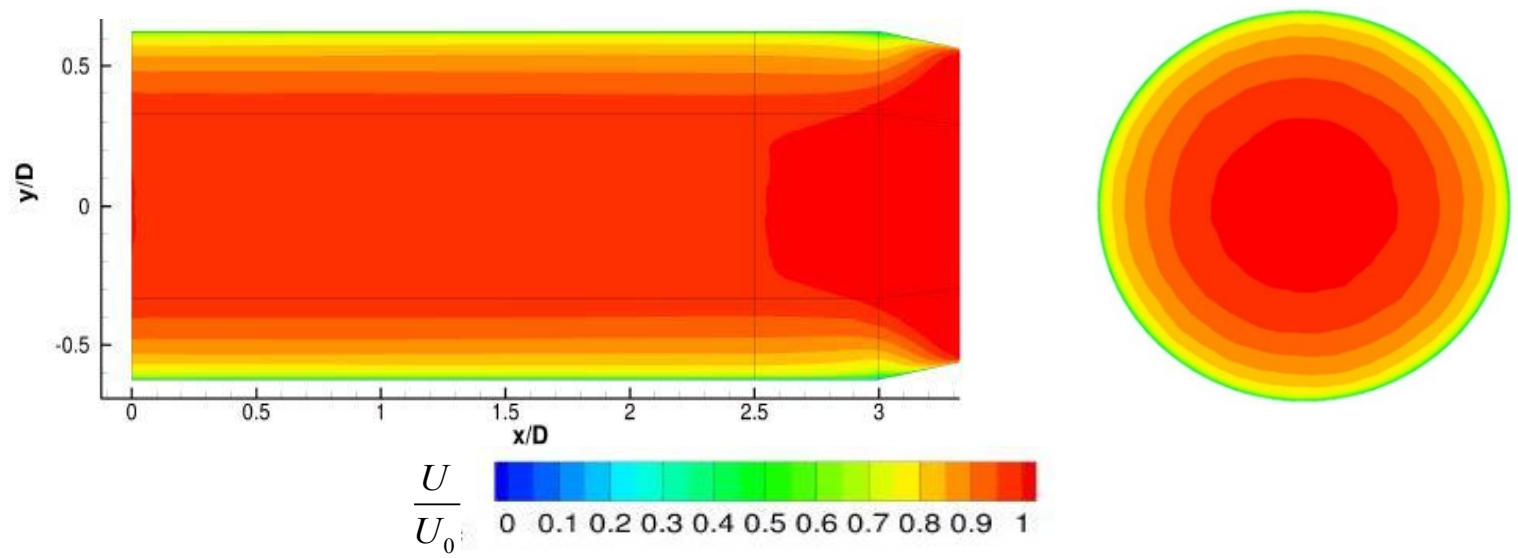

Fig. 8 LES $R^{2} M(P+G$ SGS) predictions, mean axial velocity, (left)plane $z / D=0$, (right) plane $C$ 
layer thickness, showing no sign of distortion as the solution passes through the IC/MS domain interface $(x / D=2.5)$. The cross-stream contours on the radial/azimuthal plane $C$ (MS inlet conditions) display an axisymmetric flow with the correct boundary layer thickness (see Table 2 below). Results for the Piomelli and Guerts SGS model simulation are shown in this Section, but there is little difference between the SGS models, both perform well for a zero pressure gradient boundary layer.

Quantitative assessment of the ability of the $R^{2} M$ technique to generate velocity time series with statistics that match the target data is provided in Figs. 9 and 10. In Fig. 9 the mean axial velocity profile produced for the nozzle inlet boundary condition is plotted in log-law format together with the experimental data. The LES prediction agrees well with the measured data, in both log-law region portion $\left(\sim 250<\mathrm{y}^{+}<\sim 5000\right)$ and low Reynolds number buffer region $\left(\mathrm{y}^{+}<\right.$ 100).

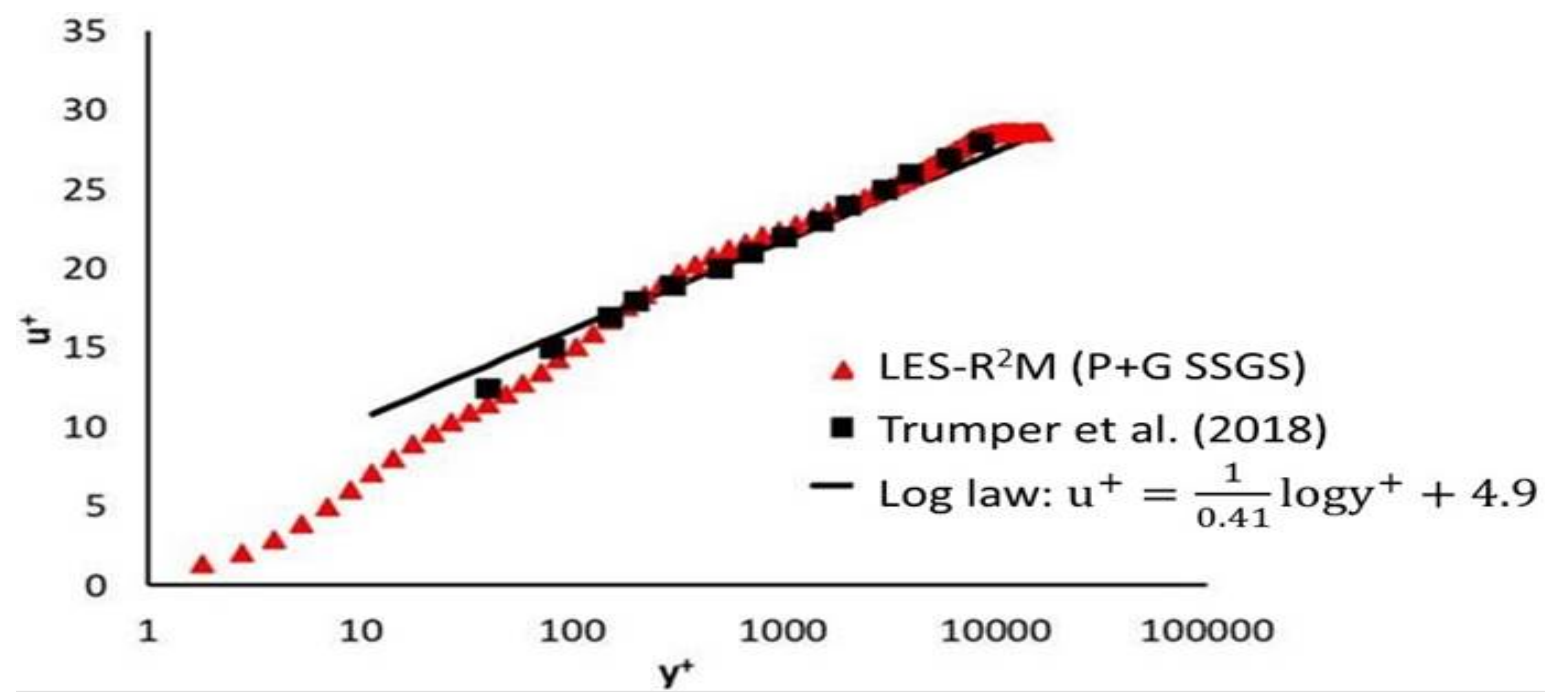

Fig. 9 LES $R^{2} M(P+G S G S)$ inlet mean axial velocity profile.

In Fig. 10 the predicted normal stress profiles are plotted along 4 radial lines $\left(0^{\circ}, 90^{\circ}, 180^{\circ}\right.$, and $270^{\circ}$ ). The excellent axisymmetry of these $2^{\text {nd }}$ order statistics demonstrates a sufficiently long averaging time. Anisotropy between streamwise, wall normal, and wall parallel components has been captured well (significantly better than in Bres at al. [6]). Peak stress values in the near wall region $(y / D<0.05)$ are slightly overpredicted for axial $(14 \%)$ and transverse $(10 \%)$ components, but underpredicted for the wall normal stress $(25 \%)$. The likely cause for these errors is the use of eqn. (9), which effectively assumes the entire profile obeys outer layer similarity. Fernholz and Finlay [30] have analysed experimental data to show that whilst this is true for $\operatorname{Re}_{\theta}>5000$ and for $y^{\prime} / \delta>0.1$, Reynolds number effects are still important for $y^{\prime} / \delta<0.1$ and inner scaling using wall units should have been used for this region. The near wall agreement could have been improved by extracting rms data using separate inner/outer similarity relations as given in Fernholz and Finlay [30]. An alternative would be to use experimental boundary layer data taken at higher Reynolds number as provided by Carlier and Stanislas [44]. 


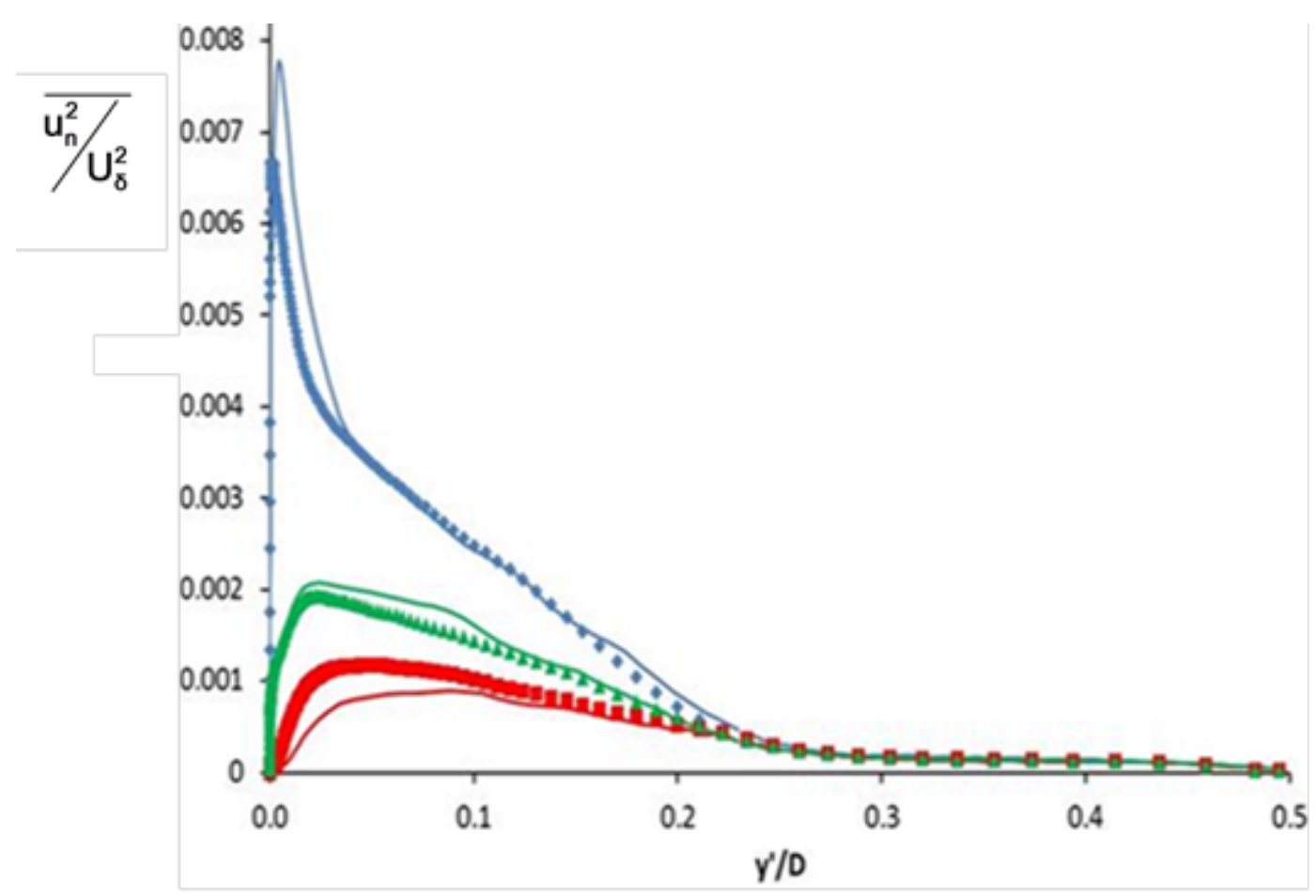

Fig. 10 LES $R^{2} M(P+G$ SGS) inlet Reynolds normal stress profiles:

blue: axial $(n=1)$, red: radial (wall normal, $n=2$ ), green: azimuthal (wall parallel, $n=3$ ) Symbols are target data

Figure 11 presents a power spectral density plot for axial turbulence fluctuations at a point in the log-law region $\left(y^{+} \sim 1000\right)$. This shows that the $R^{2} M$ technique has enabled realistic energy spectra to be created, with the expected $-5 / 3$ behaviour displayed for around 2 decades of PSD $\left(10^{-2}\right.$ to $\left.10^{-4}\right)$ before enhanced fall-off at higher frequencies where SGS viscosity is effective. There is no evidence of the recycling frequency errors reported by Morgan et al. [10]; these must be present as recycling establishes a perfect correlation between inlet and recycling planes, but this artificial frequency component is clearly in the present case at significantly lower energy than the resolved scale turbulence.

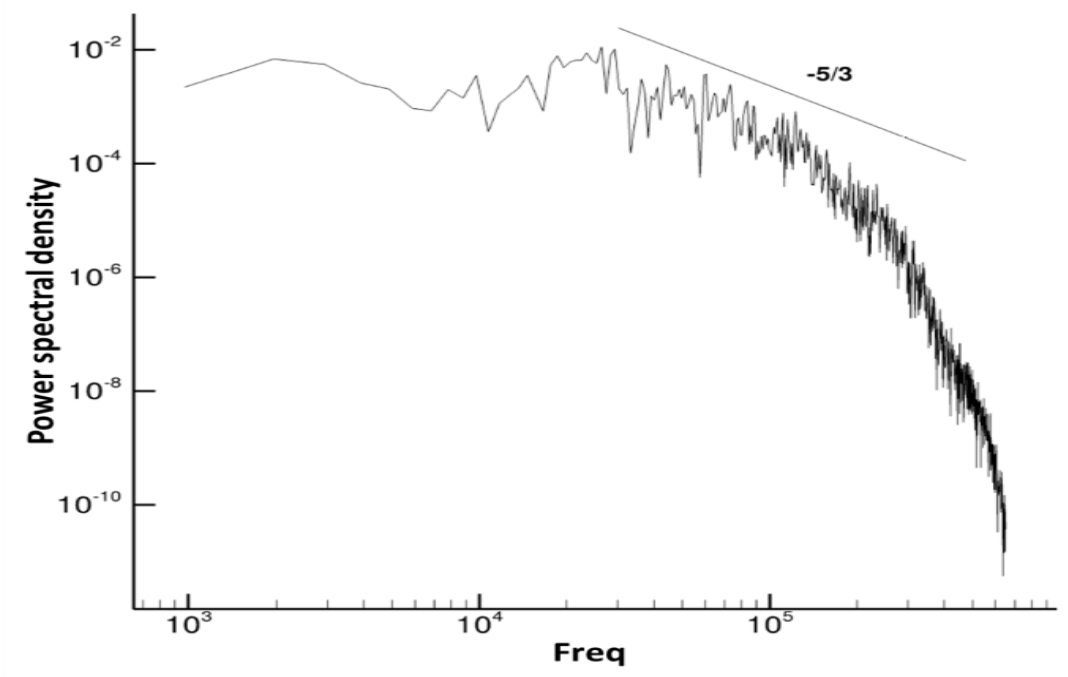

Fig. $11 \mathrm{LES} \mathrm{R}^{2} \mathrm{M}(\mathrm{P}+\mathrm{G}$ SGS) PSD at nozzle inlet. 
Finally, Table 2 provides predicted overall boundary layer parameters at nozzle inlet. Comparison between measurements and the LES solution indicates good agreement for all parameters.

Table 2: Global boundary layer parameters at nozzle inlet for NPR $=1.5$

\begin{tabular}{|c|c|c|}
\hline & Expt. Data [29] & LES R $\mathbf{R}^{\mathbf{M}}$ (P+G SGS) \\
\hline Overall boundary layer thickness $\boldsymbol{\delta}(\mathbf{m m})$ & 21.0 & 20.8 \\
\hline Momentum thickness $\boldsymbol{\theta}(\mathbf{m m})$ & 1.45 & 1.38 \\
\hline Shape factor $\left(\mathbf{H}_{\mathbf{1 2}}\right)$ & 1.33 & 1.28 \\
\hline Skin friction coefficient $\left(\mathbf{C}_{\mathrm{fi}}\right)$ & $2.38 \times 10^{-3}$ & $2.50 \times 10^{-3}$ \\
\hline Reynolds No. $\left(\mathbf{R e}_{\boldsymbol{\theta}}\right)$ & $1.88 \times 10^{4}$ & $1.79 \times 10^{4}$ \\
\hline
\end{tabular}

In summary, examination of statistical properties of the nozzle inlet time-series generated by the $\mathrm{R}^{2} \mathrm{M}$ approach - mean velocity (Fig.9 and Table 2), $2^{\text {nd }}$ moment turbulence quantities (Fig. 10), and integral length scale (Fig. 7) - shows that it has performed well, producing boundary conditions for the nozzle flow simulation that are in close accord with measured data with timeseries data over the inlet plane possess physically plausible spatial correlations (Fig. 6) and energy spectrum (Fig. 11).

\subsection{Flow Development within the Nozzle}

Figure 12 provides an illustration of the importance of an LES inlet condition method which creates physically realistic and self-consistent turbulence structures. Instantaneous axial velocity contours are displayed on 3 planes between nozzle inlet and start of convergence. Large scale energy-containing flow structures are clearly visible within the boundary layer and sustained throughout the inlet section up to the start of nozzle convergence. Without the benefit of spatial and temporal internal consistency, the simulation would lead to modification of the inlet structures - in all probability their decay - with the flow having insufficient time for fluctuations to be re-established before the acceleration region is entered. LES results are shown this time from the Smagorinski SGS model, but again the influence of the SGS model at this stage of the flow is relatively small.

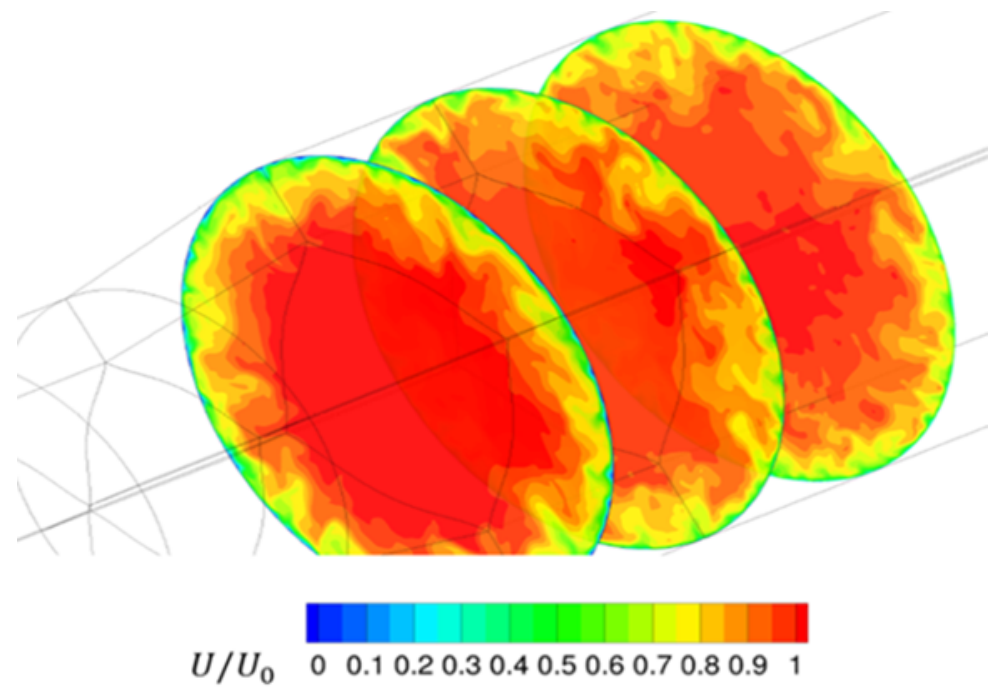

Fig. 12 Instantaneous $u$ velocity contours on 3 cross-sections between planes $C$ and $D$ LES-R²M (Smagorinski SGS) 
Figure 13 indicates how the turbulent structures develop under the influence of the nozzle acceleration. Instantaneous $U$ and $W$ velocity contours are shown on plane $z / D=0.0$ (this time from the LES-R²M (P+G SGS) simulation). Axial velocity contours (Fig.13 (top)) are shown for a region from the centre of the IC domain through to nozzle exit. The imposition of a favourable pressure gradient is seen to lead to a significant reduction in the size and amplitude of the turbulent eddies, and rapid reduction in boundary layer thickness. W contours (Fig. 13 bottom) (plotted over the entire IC and MS domains) characterise resolved scale large vortical structures revealed by regions of alternating $\mathrm{W}$-velocity sign. These structures are angled away from the wall as observed in boundary layer measurements and are stretched longitudinally as they pass through the convergence, reflecting the Warnack and Fernholz [19] observation of increased streamwise integral scales. Unlike the $U$-structures, the W-structures persist to nozzle exit.
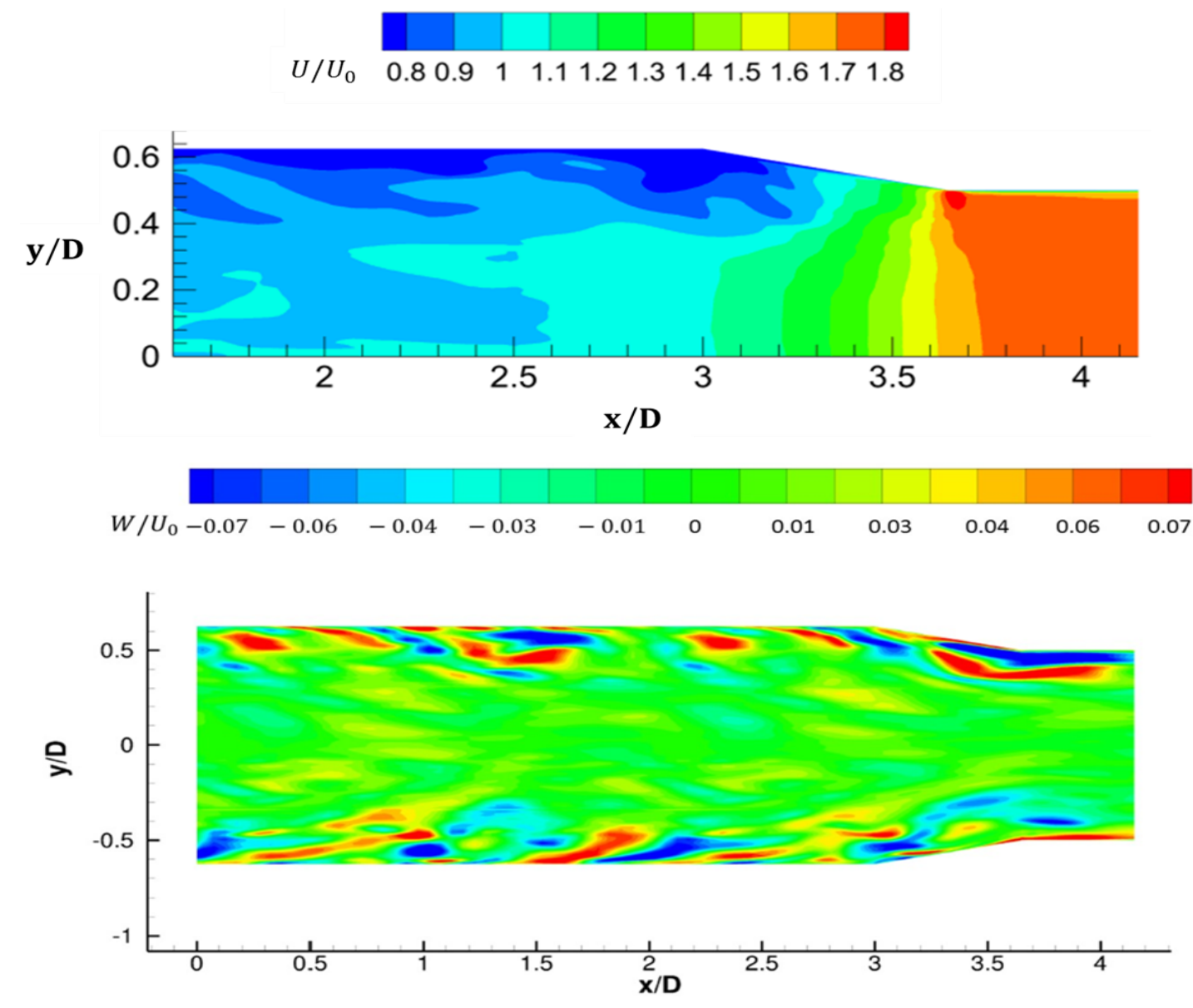

Fig. 13: LES $R^{2} M(P+G$ SGS) instantaneous $U$ (top) and $W$ (bottom) on plane $z / D=0$

The predicted time-averaged axial velocity field within the nozzle on a vertical diametral plane $(z / D=0)$ is displayed in Fig. 14, again for the P+G SGS model. Flow acceleration already starts to appear $\sim 0.4 \mathrm{D}$ upstream of the convergence, with the thick inlet boundary layer drastically reducing in thickness by half-way through the convergence section. Evidence of a much thinner wall boundary layer re-appears in the nozzle exit parallel extension growing from the convergence/parallel extension corner. The contour plot also shows a small local region of acceleration/deceleration associated with flow curvature caused by the same corner; the effect of this is very significant and will be discussed further below. 


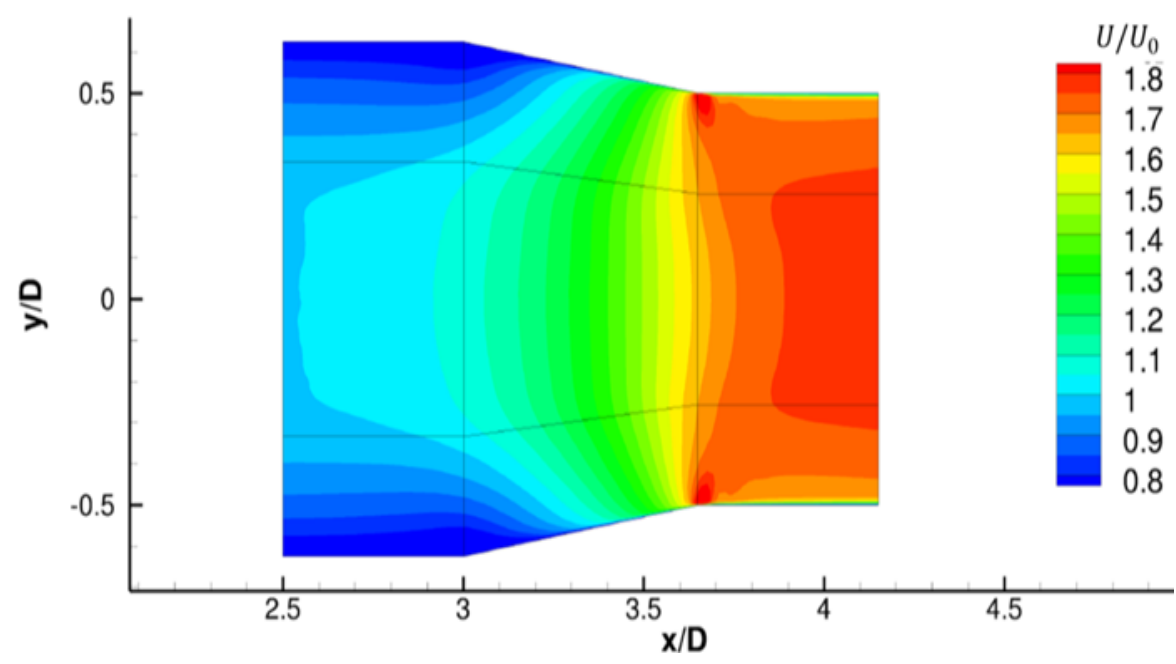

Fig. 14 LES $R^{2} M(P+G S G S)$ predicted time-mean axial velocity on $z / D=0$ plane

It is in the convergent nozzle section where the favourable pressure gradient will exert a strong influence on the turbulence structure; the normal stress anisotropy may depart significantly from its inlet equilibrium structure (Fig. 10). Evidence of this is revealed by in Fig. 15 which displays turbulence statistics at start (plane D) and end (plane E) of the convergence section; results with both SGS models are presented. Note that profiles are nondimensionalised using local boundary layer edge freestream velocity and boundary layer thickness to allow easier comparison, since acceleration/recovery processes change boundary layer size significantly $-\delta=21 \mathrm{~mm}($ at $\mathrm{C}$ ), $=0.3 \mathrm{~mm}$ (close to plane $\mathrm{E}$ ), and $=12 \mathrm{~mm}$ (at $\mathrm{F}$ ).

The most noticeable deviation from equilibrium turbulence characteristics at the start of convergence (Fig. 15a) is an increase in the near-wall $\overline{u^{2}}$ peak value compared to the equilibrium level (by $\sim 85 \%$, Figs. 10 and 15a). This response is consistent with the measurements of Bourassa and Thomas [44] for a highly accelerated turbulent boundary layer in a plane convergent duct. Their data showed that as the flow approached the convergence the near wall axial stress increased by $\sim 70 \%$, agreeing well with Fig.15a. The explanation is that a favourable pressure gradient is also induced in the constant area duct upstream of convergence (see Fig. 14). Although in this region $\mathrm{K}$ remains comparatively small (here as in the Bourassa and Thomas [44] case only $\sim 1.0 \times 10^{-6}$ ), thinning of the boundary layer is still observed. The extra strain rate associated with this then leads to enhanced turbulence production of $\overline{u^{2}}$. Fig. 15a shows this effect is restricted to the near wall region (as also in Bourassa and Thomas [43]). Both SGS models produce similar $\overline{u^{2}}$ profiles; the models differ only in the response of the wall parallel stress $\overline{w^{2}}$, where the Smagorinski prediction shows an essentially unchanged $\overline{w^{2}}$ peak, whereas the $P+G$ model produces an extra peak very close to the wall.

The flow experiences much stronger acceleration further downstream in the convergence section. The expected re-laminarisation process will lead to diminished turbulence levels. The strength of reduction due to flow acceleration may be estimated by examining the Piomelli and Yuan [15] data, where the peak K value $\left(4.0 \times 10^{-6}\right)$ was similar to that in the current nozzle flow. Comparison of Figs. 10 and 15b shows that non-dimensional turbulence levels have certainly decreased in the nozzle flow. More detailed comparison, however, reveals significant differences to the simulation results of [15]. Using peak turbulence levels as a measure, the present flow shows maximum values of $\overline{u^{2}} / \overline{v^{2}} / \overline{w^{2}}$ have reduced to around $20 \% / 75 \% / 80 \%$ of 
their inlet values. This is in stark contrast to behaviour reported in [15], where these numbers were around $95 \% / 15 \% / 15 \%$. Individual normal stresses in the current flow are clearly behaving quite differently than expected purely due to favourable pressure gradient effects. The nozzle flow predictions are essentially the same with both SGS models, although a slightly lower $\overline{u^{2}}$ peak and higher $\overline{w^{2}}$ peak are observed in the Smagorinski prediction.
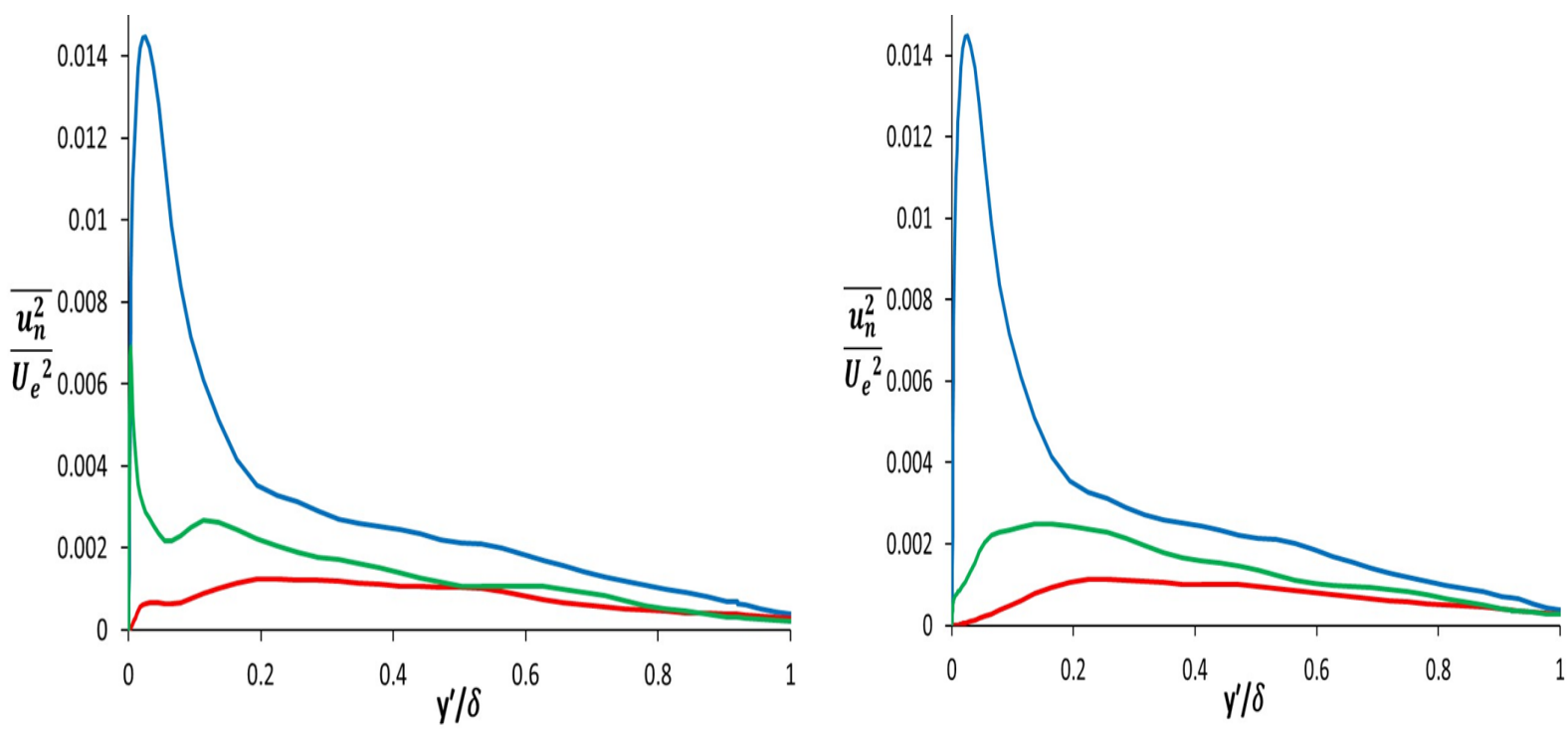

(a)
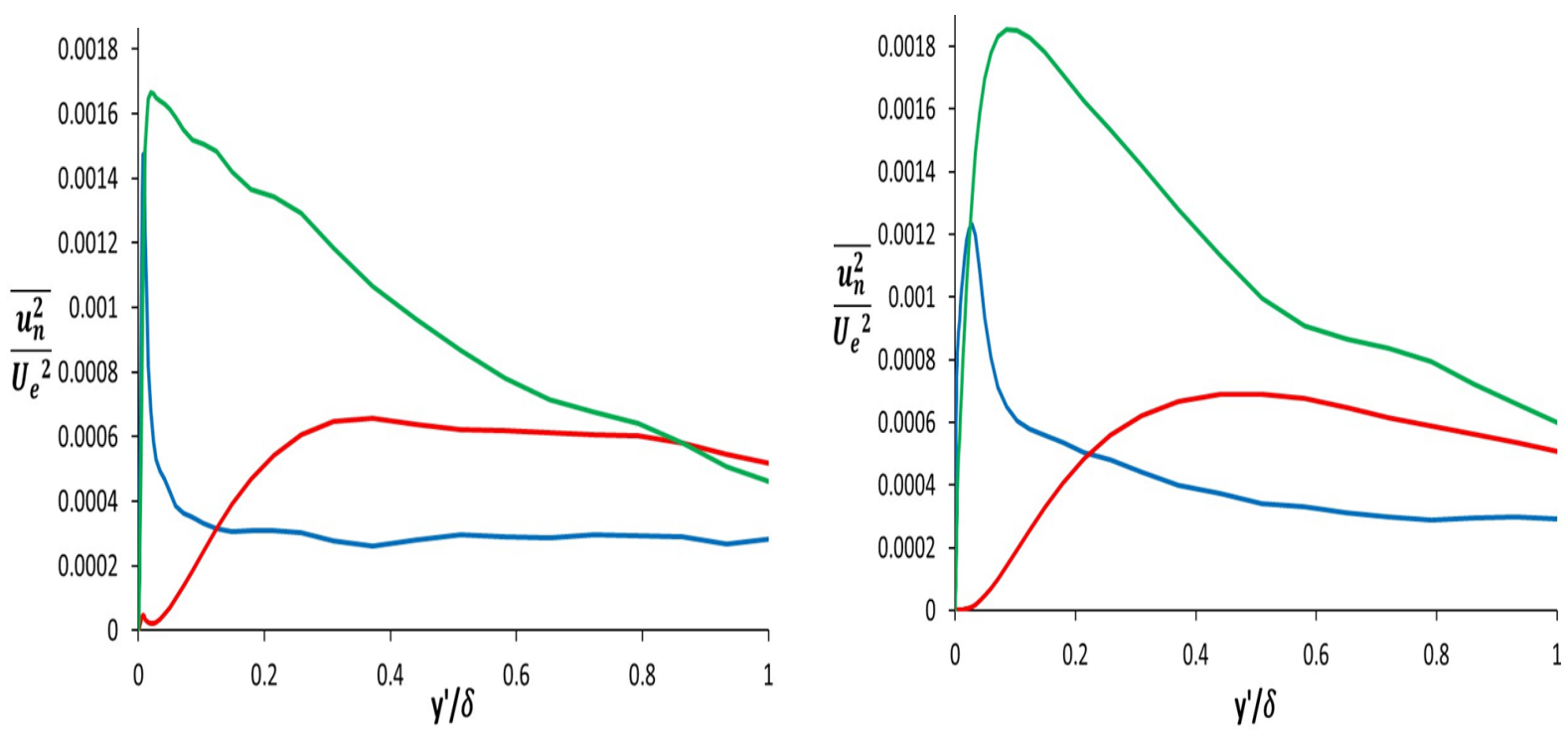

(b)

Fig. 15 LES $R^{2} M$ with $P+G$ (left) and Smagorinski (right) SGS models Reynolds normal stress profiles, $z / D=0$ plane, (a) plane $D$, (b) plane $E$,

Blue $-\overline{u^{2}}$ axial $(\mathrm{n}=1)$, red $-\overline{v^{2}}$ radial (wall normal, $\left.\mathrm{n}=2\right)$, green $-\overline{w^{2}}$ azimuthal (wall parallel, $\mathrm{n}=3$ ) 
It is clear that some other factor in addition to bulk flow acceleration is active in the current flow. The study reported in [15] focussed on the isolated imposition of a favourable pressure gradient on a boundary layer developing on a flat surface, whereas the present flow has a different geometrical arrangement and in particular includes a corner between convergence and parallel extension sections. It is possible - as noted in [29] - that corner flow effects have exerted a strong influence on the near-wall flow and turbulence development. The observations made on Fig. 15b are consistent with earlier comments where attention was drawn to a small region of high velocity in the corner location (the near wall region of high velocity at $x / D=3.65$ in Fig. 14). This suggests that near-wall flow development has been modified both upstream and downstream of the corner. The upstream effect is shown in Fig.16, which presents a predicted time-averaged axial velocity profile just upstream of the corner location (from both SGS models and normalised by $U_{\mathrm{CL}}$. . A local maximum very close to the wall and a wall-jet like profile in the region within $10 \%$ of the wall have appeared. The near wall flow structure no longer resembles a boundary layer-like shape. Both SGS models display the same flow feature; the P+G SGS model indicates a substantially stronger acceleration, whilst the Smagorinski SGS result has a similar profile shape but a smaller peak velocity.

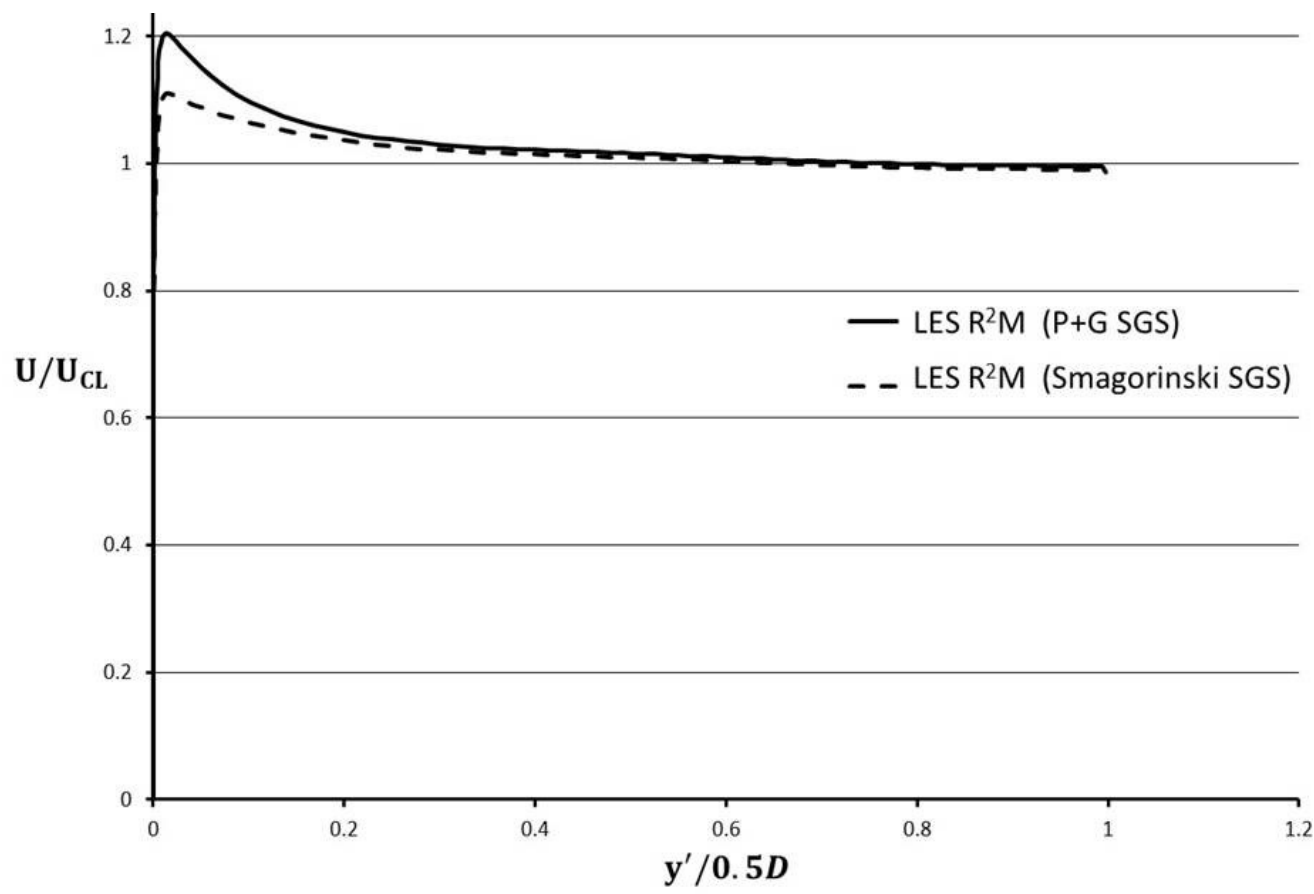

Fig. 16 LES $R^{2} M$ predicted mean axial velocity profiles near convergence end

To examine effects downstream of the corner, Fig.17 presents a zoomed-in view of a region very close to the wall and immediately downstream (radially only $0.004 \mathrm{D}(\sim 0.25 \mathrm{~mm})$ and axially only $0.2 \mathrm{D}(\sim 12 \mathrm{~mm})$. The $P+G$ SGS model reveals the appearance of a small recirculation region, whereas in the Smagorinski SGS simulation no negative velocity is apparent, rather just enhanced growth of the near wall low velocity region. In fact, closer examination revealed a single row of cells immediately next to the wall (the region $x / D=3.65-3.7$ ) does contain very small velocities $\left(U / U_{0}<1 \%\right)$. Further inspection of the instantaneous Smagorinsky SGS timeseries in this region showed this was characterised by generally positive axial velocity with occasional bursts of negative velocity. 

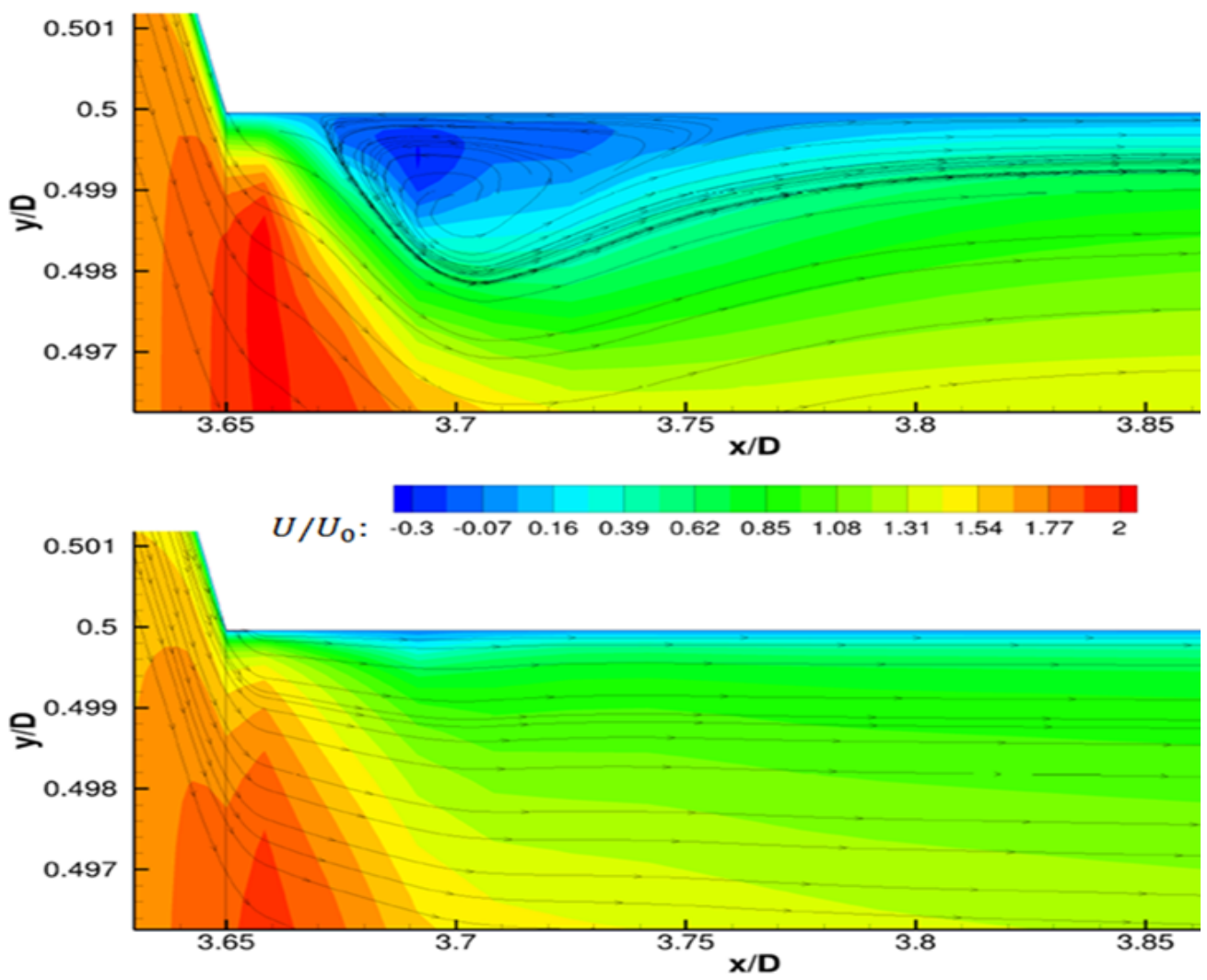

Fig. 17 Near wall mean axial velocity contours, start of nozzle exit parallel section, (top) LES R ${ }^{2} M$ (P+G SGS), (bottom) LES R²M (Smagorinski SGS)

Clearly, the Smagorinsky solution avoids time-mean separation because its SGS eddy viscosity is sufficiently larger than in the P+G SGS model to promote more momentum diffusion into the near-wall region just after the sharp corner. Both flow patterns in Fig.17 are consistent with the presence of a blockage, which accelerates (see Fig. 16) and deflects the upstream bulk flow radially inwards. The backflow region in the P+G SGS simulation causes stronger flow deviation than the weaker blockage in the Smagorinski SGS simulation and is consistent with the higher peak velocity in Fig.16. The differing profile shapes in Fig.16 imply stronger strain rates with the $P+G$ SGS model and hence the cause of slightly different turbulence profiles between SGS model predictions at plane E e.g. higher $\overline{u^{2}}$ peak with the P+G SGS model

These velocity differences inevitably cause different turbulence development in the parallel extension section, as illustrated in Fig.18 via contours of resolved axial normal stress (for a similar near wall zone as Fig. 17). The higher strain rates associated with the presence of a recirculation zone in the $P+G$ SGS model generate much higher levels of turbulence (and much closer to the wall) than in the Smagorinsky SGS simulation. The $P+G$ peak value is in the shear layer on the edge of the recirculation zone, whereas in the Smagorinski calculation the turbulence level is highest in the central region of the thickening boundary layer and increases gradually downstream, reaching a peak close to nozzle exit. A high 'spot' of turbulence exists close to the wall just downstream of the corner in the Smagorinski SGS results; this is evidence of the transitory separation described above. It is important to note the contour scale difference in Fig. 18, the peak value with the $P+G$ SGS model is an order of magnitude greater than predicted by the Smagorinski SGS. 

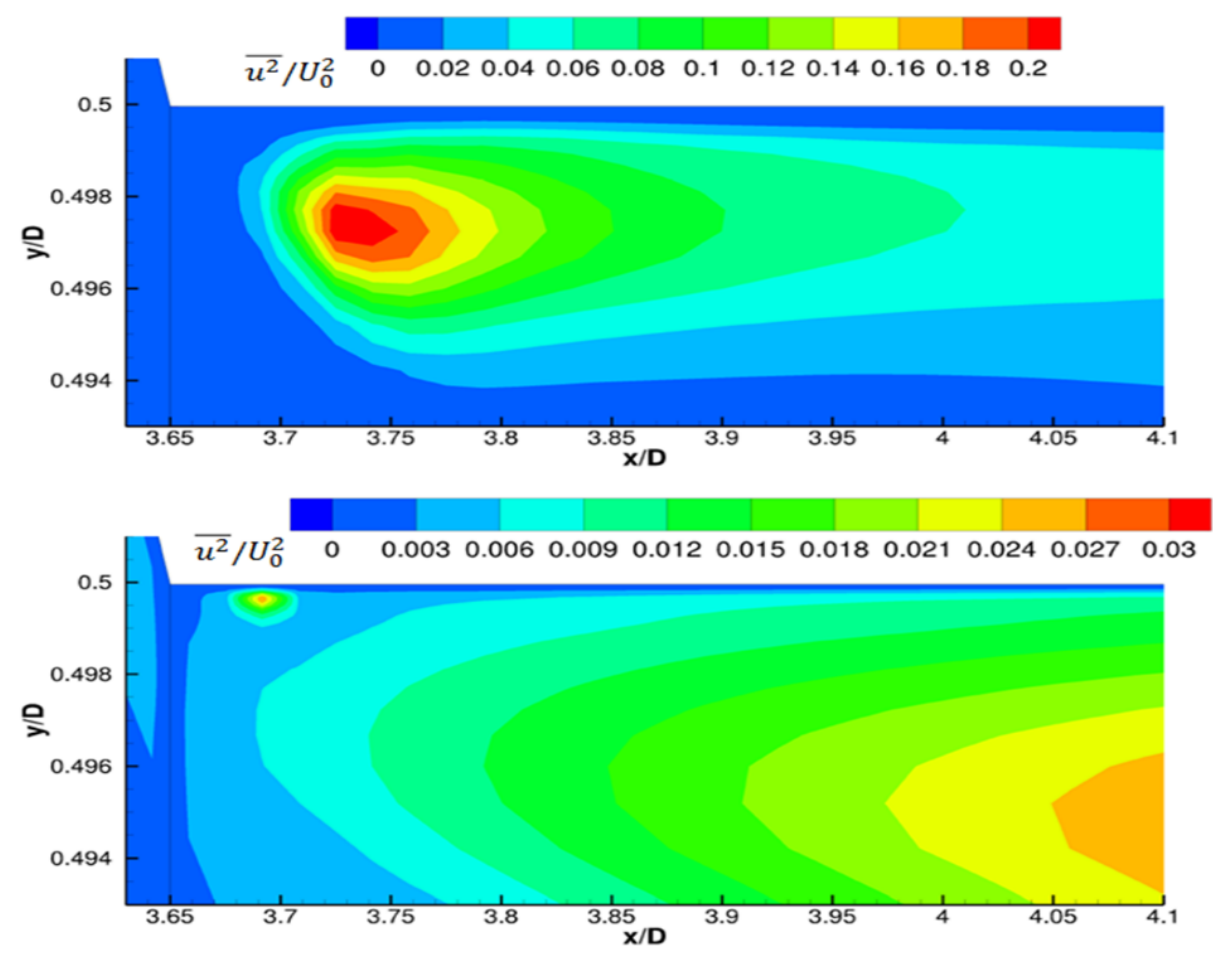

Fig. 18 Near-wall resolved axial normal stress, start of nozzle exit parallel section P+G SGS model (top); Smagorinski SGS model (bottom)

Given the large differences indicated in Fig.18, it is inevitable that predicted turbulence conditions at nozzle exit differ markedly between the two SGS models (Fig. 19).
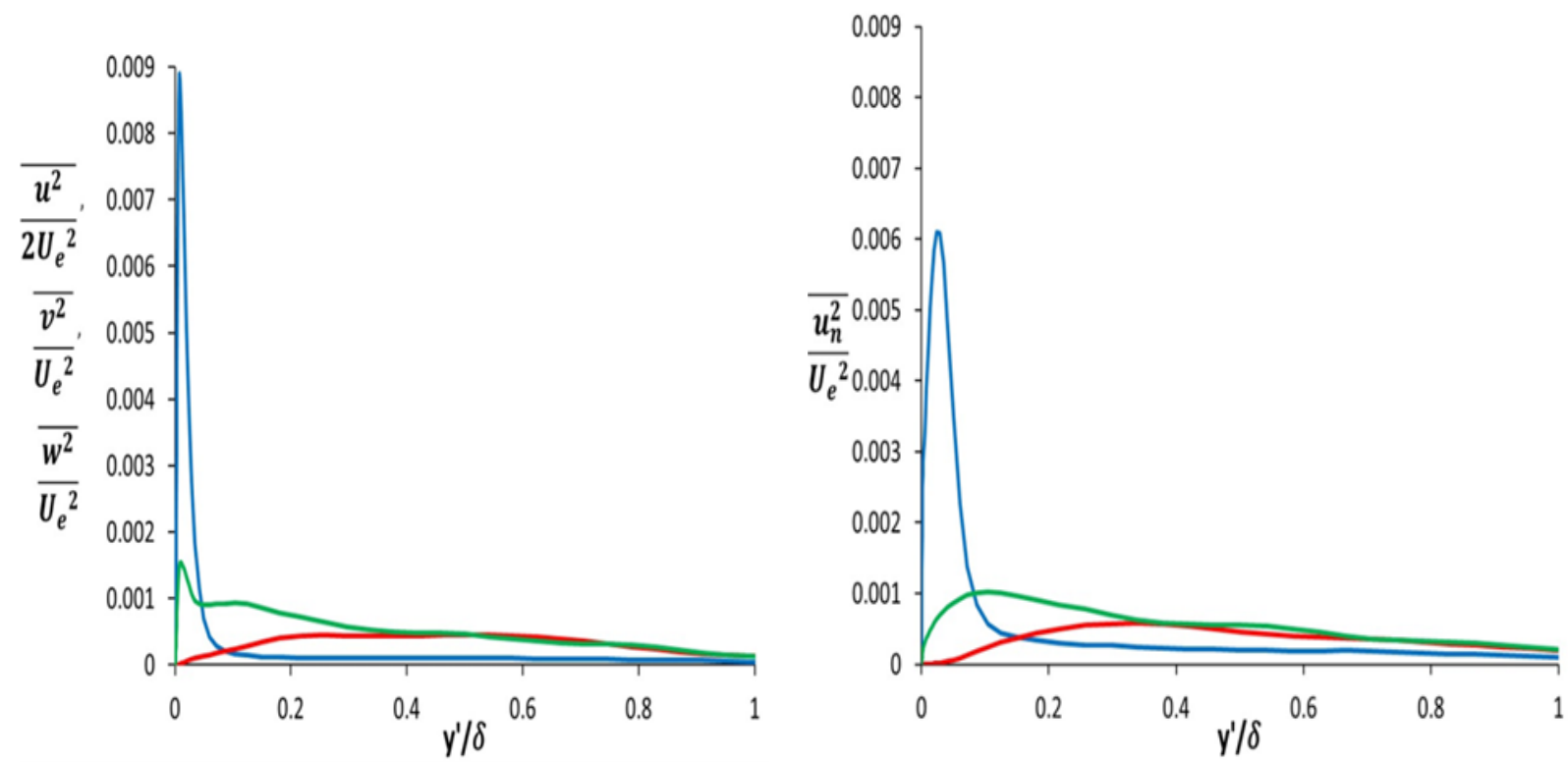

Fig. 19 LES $R^{2} M$ predictions of Reynolds normal stress profiles at nozzle exit Left - P+G GS model, Right - Smagorinski SGS model Blue $-\overline{u^{2}}$ axial $(\mathrm{n}=1)$, red $-\overline{v^{2}}$ radial (wall normal $\left.\mathrm{n}=2\right)$, green $-\overline{w^{2}}$ azimuthal (wall parallel, $\mathrm{n}=3$ ) 
For both SGS models the axial $\overline{u^{2}}$ stress has returned to being the largest, although peak values are quite different between the two models (NB $\overline{u^{2}} / 2 U_{e}^{2}$ is plotted for this model). In the Smagorinsky SGS simulation the peak axial stress is almost the same as in the inlet boundary layer; in the P+G SGS simulation, however, it is twice that in the inlet profile. For both SGS models $\overline{v^{2}}$ and $\overline{w^{2}}$ stresses have essentially recovered to close to their inlet profile levels, although the extra near wall $\overline{w^{2}}$ peak noted for the $P+G$ model in Fig. 15a has now returned. The development of turbulence in the parallel extension is clearly very different between the two SGS model, only comparison with nozzle exit measurements can determine which captures the flow physics better, and this is addressed in Section 4.3.

\subsection{Flow Conditions at Nozzle Exit and Comparison with Measurements}

Table 3 first presents measured and predicted global boundary layer parameters at nozzle exit; at this level of integrated mean velocity properties, both SGS models provide solutions close to the measured data.

Table 3: Global Boundary layer Parameters at Nozzle Exit

\begin{tabular}{|l|c|c|c|}
\hline & $\begin{array}{c}\text { Expts. } \\
{[29]}\end{array}$ & $\begin{array}{c}\text { LES R } \\
\text { Smagorinski SGS }\end{array}$ & $\begin{array}{c}\text { LES R } \\
\text { P+G SGS }\end{array}$ \\
\hline Momentum thickness $\boldsymbol{\theta}(\mathbf{m m})$ & 0.362 & 0.344 & 0.347 \\
\hline Shape factor $\left(\mathbf{H}_{\mathbf{1 2}}\right)$ & 1.22 & 1.24 & 1.22 \\
\hline Reynolds number $\left(\mathbf{R e}_{\boldsymbol{\theta}}\right)$ & 7494 & 6800 & 6760 \\
\hline
\end{tabular}

Exit profiles for the mean velocity (in Clauser-chart format) are examined in more detail in Fig. 20, which compares experimental data, LES results from both SGS models and results from a low Re Launder-Sharma RANS prediction.

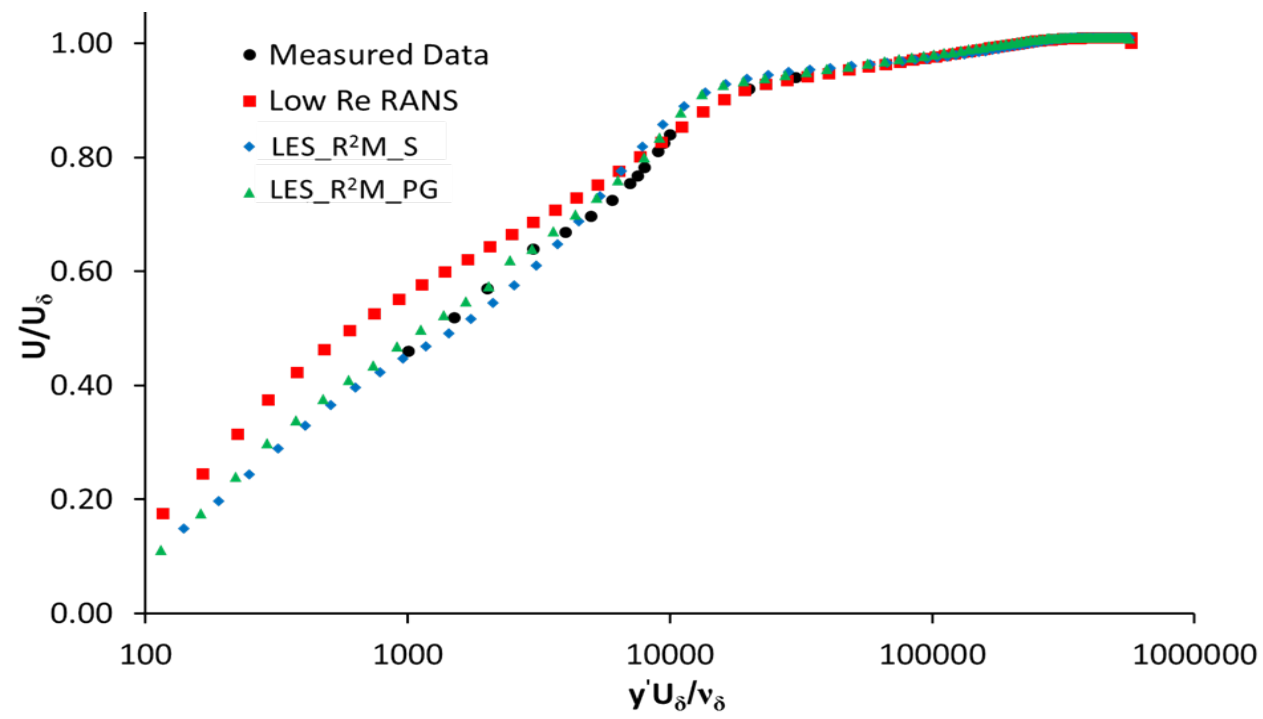

Fig. 20 Predictions of nozzle exit mean axial velocity profile

Both LES predictions show close agreement with experimental data, with the $P+G$ model slightly better close to the wall; a definite improvement is seen when LES is compared to low $\operatorname{Re}$ 
RANS predictions. Based on mean velocity accuracy alone, it is difficult to distinguish between the two SGS models.

A quite different picture emerges when exit turbulence profiles are examined. Fig. 21 compares predicted axial rms using both SGS models against nozzle exit LDA measurements.
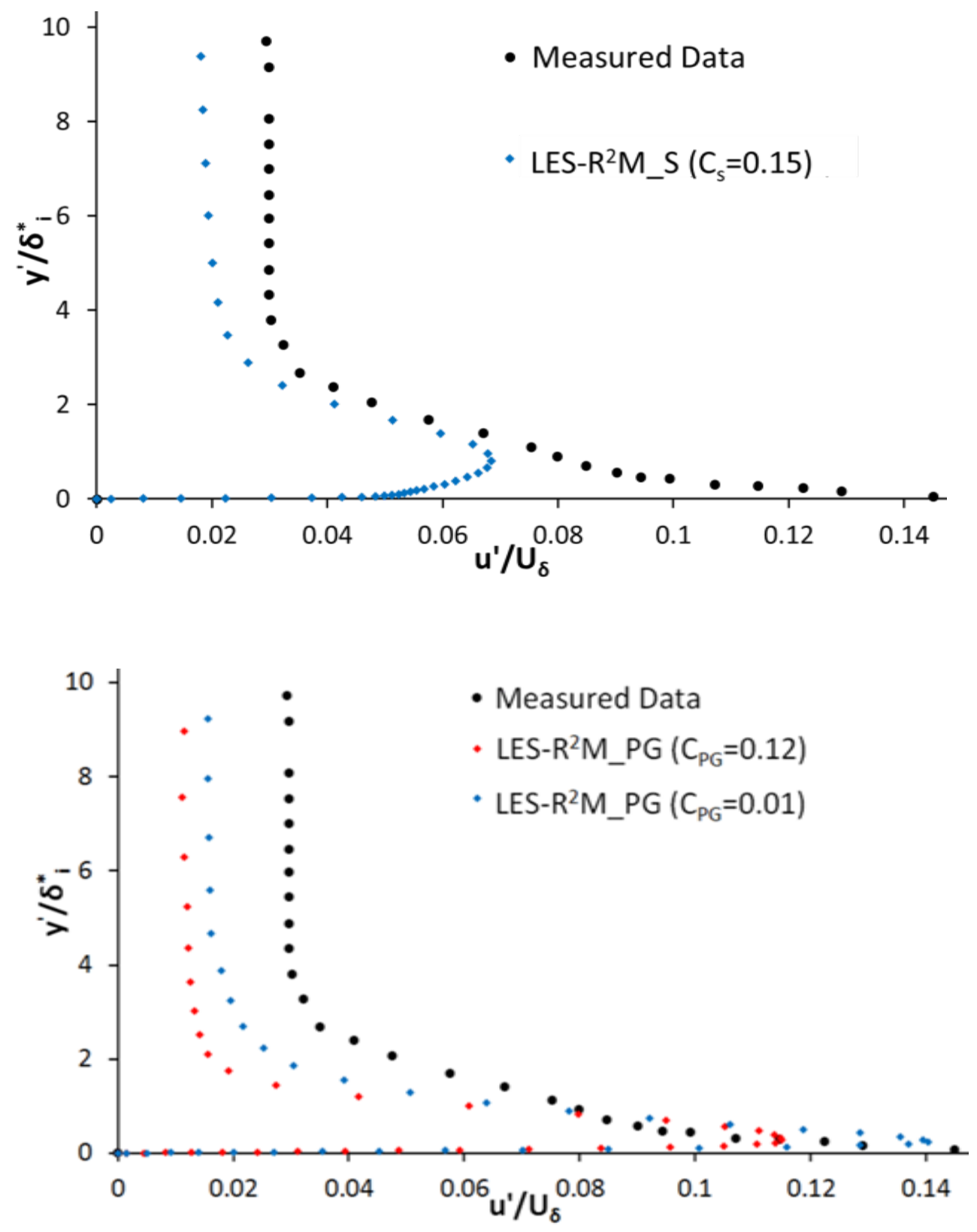

Fig. 21: LES $R^{2} M$ predicted nozzle exit axial turbulence intensity compared to measurements Top: Smagorinski SGS model, Bottom: Piomelli and Geurts SGS model

For the Smagorinsky model the measurements are significantly under-predicted - a peak value less than half that measured. The location of the peak stress is also substantially further away from the wall than in the measured data, with the profile from the simulation showing a much more rounded shape. All these aspects are considerably improved when the Piomelli and 
Guerts model results are examined. Data for two values of the constant $\mathrm{C}_{\mathrm{PG}}$ are shown, covering the entire range explored during the optimisation study reported in Section 4.1. Both values show superior performance compared to the Smagorinsky results. The value of $\mathrm{C}_{P G}$ identified by optimising the prediction of boundary layer inlet integral length scale returns very close agreement with the LDA measurements, predicting the measured peak value, its location and the general profile shape well. The sensitivity to $C_{P G}$ is in fact not very great and the different profile shapes between the SGS models is entirely consistent with the observations made when discussing Fig. 18. It is clear that the corner effects have played a dominant role in determining the exit turbulence characteristics. It is also notable that the level of turbulence in the core flow outside the boundary layer is not well predicted by any of the simulations. The probable cause for this is that when the target data for the nozzle inlet conditions were specified, emphasis was placed primarily on the high turbulence region inside the boundary layer. If the actual turbulence in the outer core flow of the Trumper et al. [29] experiment had been known, a better fit to this part of the profile might have been achieved.

\subsection{CONCLUSIONS}

- Review of the available literature showed that the nozzle component of LES-based CFD approaches proposed to date for aeroengine nozzle/exhaust plume analysis had not been appropriately validated against relevant experimental data.

- The data of Trumper et al. [29] were identified as a suitable validation test case. The nozzle geometry included strong acceleration - addressing the issue of possible boundary layer re-laminarisation - also a short parallel nozzle exit extension - enabling boundary layer recovery. This data was thus considered to represent a challenging test case. Measurements were conducted at relevant values of Reynolds/Mach numbers and at nozzle inlet/exit, allowing LES inlet condition specification and methodology assessment.

- A rescaling/recycling method for LES inlet condition generation was adopted and was shown to be successful in creating spatially- and temporally-correlated turbulent structures at nozzle inlet whose statistical properties matched measured $1^{\text {st }}$ and $2^{\text {nd }}$ moment profiles as well as integral length scale and energy spectra.

- The SGS constant in the mesh-independent Piomelli and Geurts [33] SGS model was calibrated to provide optimum prediction of boundary layer integral length scale measurements.

- This model then demonstrated overall superior performance compared to the standard Smagorinsky model. Both models predicted the exit mean velocity profile accurately, but comparison with measured exit turbulence characteristics revealed a considerable improvement with the Piomelli and Guerts model. Both amplitude and location of the peak stress and the overall profile shape were better predicted.

- The root cause of the improvement was the ability of the simulation based on the Piomelli and Geurts [33] SGS model to resolve a small separation region induced at the internal corner between convergent and parallel nozzle wall sections.; this was not present in the simulation using the Smagorinski model.

- Accurate prediction of near-field phenomena is essential for such important parameters as potential core length and high frequency noise generation in the early part of the jet free shear layer. The first step in precise capture of near-field development is an accurate description of nozzle exit flow and turbulence characteristics The validated LES methodology presented here has demonstrated good performance in this regard and is recommended for application to the more complex nozzle shapes found in practice. It 
represents a superior approach for capture of nozzle exit conditions and should be applied in jet plume aerodynamic and aeroacoustic simulations to establish the level of improvement it can deliver.

\section{FUNDING SOURCES}

This research was supported by a UK Higher Education ORS award and a Loughborough University Departmental studentship for the first author.

\section{REFERENCES}

[1] Kenzakowski, D., PAPP, J., DASH, S. Modelling turbulence anisotropy for jet noise prediction, AIAA 2002-0076, 40 ${ }^{\text {th }}$ Aerospace Sciences Meeting, Reno, NV, USA, January 2002.

[2] Georgiadis, N. J. \& DeBonis, J. R. Navier-Stokes analysis methods for turbulent jet flows with application to aircraft exhaust nozzles, Prog in Aerospace Sci., Nos. 5-6, 42, 2006, pp. 377-418.

[3] DeBonis, J.R. Progress toward Large Eddy Simulations for prediction of realistic nozzle systems," AIAA Journal of Propulsion and Power, No. 5, 25, 2007, pp. 971-980.

[4] Karabasov, S. A., Afsar, M. Z., Hynes, T. P., Dowling, A. P., McMullan, W. A., Pokora, C. D., Page, G. J., McGUIRK, J. J. Jet noise: acoustic analogy informed by Large Eddy Simulation, AIAA Journal, No. 7, 48, 2010, pp. 1312-1325.

[5] WANG, P. C. \& McGUIRK, J. J. Large Eddy Simulation of supersonic jet plumes from rectangular con-di nozzles, International Journal of Heat and Fluid Flow, Oct. 43, 2013, pp. 62-73.

[6] Bres, G. A., Jaunet, V., Le Rallic, M., Jordan, P., Colonius, T., Lele, S. K. Large Eddy Simulation for jet noise: the importance of getting the boundary layer right," AIAA-2015-2535, 21st AIAA/CEAS Aeroacoustics Conf., June 2015.

[7] Chung, Y.M. \& SUNG, H.J. Comparative study of inflow conditions for spatially evolving simulation, AlAA Journal, No.2, 35, 1997, pp. 269-274.

[8] TABOR, G.R. \& BABA-AHMADI, M.H. Inlet conditions for Large Eddy Simulation: a review, Computers and Fluids, No. 4, 39, 2010, pp. 553-567.

[9] Wu, X. Inflow turbulence generation methods, Ann. Rev. Fluid Mech., January 49, 2017, pp. $23-49$.

[10]Morgan, B., LARSSON, J., KAWAI, S., LELE, S. K. Improving low frequency characteristics of recycling/rescaling inflow turbulence generation, AIAA Journal, No. 3, 49, 2011, pp. 582-597.

[11]LUND, T. S., WU, X., SqUIRES, K. D. Generation of turbulent inflow data for spatially developing boundary layer simulations, Journal of Computational Physics, No. 2, 140,1998, pp. 233-258.

[12] LIU, K. \& PLETCHER, R. H. Inflow conditions for LES of turbulent boundary layers: a dynamic recycling procedure, Journal of Computational Physics, No. 1, 219, 2006, pp. 1-6.

[13]Araya, G., Castillo, L., Meneveau, C., Jansen, K. A dynamic multi-scale approach for turbulent inflow boundary conditions in spatially developing flows, Jnl of Fluid Mech., March 670, 2011, pp. 581-605.

[14] NIKITIN, N. Spatial periodicity of spatially evolving turbulent flow caused by inflow boundary conditions, Physics of Fluids, No. 9, 19, 2007, pp. 091703.

[15] PIOMELLI, U. \& YUAN, J. Numerical simulations of spatially developing, accelerating boundary layers, Physics of Fluids, No. 10, 25, 2013, pp. 10.1063.

[16]NARAsimHA, R. \& SREenivasan, K.R. Re-laminarisation in highly accelerated turbulent boundary layers, Journal of Fluid Mechanics, No. 3, 61, 1973, pp. 417-447.

[17] JONES, W. P. \& LAUNDER, B. E. The prediction of laminarisation with a two equation model of turbulence, Int. Journal of Heat and Mass Transfer, No. 2, 15, 1972, pp. 301-314.

[18]YANG, X. \& TUCKER, P.G. Assessment of turbulence model performance: severe acceleration with large integral scales, Computers and Fluids, March 126, 2016, pp. 81-191. 
[19] WaRnack, D. \& FERnholz, H. H. The effects of a favourable pressure gradient and of the Reynolds number on an incompressible axisymmetric turbulent boundary layer - Part 1: the turbulent boundary layer, Journal of Fluid Mechanics, March 359, 998a, pp. 329 - 356.

[20]WARNACK, D. \& FERNHOLZ, H. H. The effects of a favourable pressure gradient and of the Reynolds number on an incompressible axisymmetric turbulent boundary layer - Part 2: the boundary layer with re-laminarisation, Journal of Fluid Mechanics, March 359,1998b, pp. 357-381.

[21]PIERCE, C.D. \& MOIN, P. Method for generating equilibrium swirling inflow conditions, AIAA Journal, No. 7, 36, 1998, pp.1325-1327.

[22] XIAO, F., DIANAT, M., MCGUIRK, J.J. An LES turbulent inflow generator using a recycling and rescaling method, Flow, Turbulence and Combustion, No. 3, 98, 2017, pp. 663-695.

[23] LI, J., PAGE, G. J., McGUIRK, J. J. RANS/LES modelling for aerodynamic coupling of outlet guide vane and pre-diffuser flows, AIAA Journal, No. 1, 53, 2015, pp. 678-691.

[24]BOGEY, C. \& MARSDEN, O. Identification of the effects of the nozzle exit boundary layer thickness and its corresponding Reynolds number in initially highly disturbed subsonic jets, Physics of Fluids, No.5, 25, 2013, pp. 055106.

[25]Pokora, C. D., McMullan, W. A., PAGE, G. J., McGuiRK, J. J. Influence of a numerical boundary layer trip on spatio-temporal correlations within LES of a subsonic jet, AIAA-2011-2920, 17th AIAA/CEAS Aeroacoustics Conference, Portland, Oregon, USA, June 2011.

[26] UzUn, A., JonghoON, B., HussainI, M. Y. High-fidelity numerical simulation of a chevron nozzle jet flow, International Journal of Aeroacoustics, October 10, 2011, pp. 531-564.

[27]Fosso-Pouangue, A., Sanjose, M., Moreau, S, Daviller, G., Deniau, H. Subsonic jet noise simulations with using both structured and unstructured grids, AIAA Journal, No. 1, 53, pp. 55-69.

[28]Bres, G. A., Jordan, P., Jaunet, V., Le Rallic, M., Cavalier, A. V. G., Towne, A., Lele, S. K., Colonius, T., SCHMIDT, O. T. Importance of the nozzle exit boundary layer state in subsonic turbulent jets, Journal of Fluid Mechanics, September 851,2018, pp. 83-124.

[29] Trumper, M.T., Behrouzi, P., McGuiRk, J. J. Influence of nozzle exit conditions on the near-field development of high subsonic and underexpanded axisymmetric jets, Aerospace, March 5, 2018, pp.35-60.

[30] FERNHOLZ, H. H. \& FINLEY, P. J. The incompressible zero-pressure gradient turbulent boundary layer: an assessment of the data, Progress in Aerospace Sciences, No. 4, 32, 1996, pp. 245-311.

[31] YOUNG, A. D. Boundary Layers, BSP Professional Books, AIAA, Reston, VA, USA, 1989.

[32] SMAGORINSKY, J. General circulation experiments with the primitive equations, I: the basic experiment, Monthly Weather Review, March 91, 1963, pp. 99-164.

[33]VAN DRIESt, E.R. On turbulent flow near a wall, Jnl. of Aero. Soc., No. 11, 23, 1956, pp. 1007-1011.

[34] PiomelLI, U. \& GeURTS, B. J. A grid independent length scale for Large Eddy Simulations of wall bounded flows, Proc. Of 8th Int. Symp. on Eng. Turbulence Modelling \& Measurements - (Eds. M. A. Leschziner, P. Bontoux, B. J. Geurts, B. E. Launder \& C. Tropea), March 2010, pp. 226-231.

[35] Piomelli, U., Rouhi, A., Geurts, B. J. A grid-independent length scale for large eddy simulations, Journal of Fluid Mechanics, March 766, 2015, pp. 499-527.

[36]PAGE, G. J., LI, Q., MCGUIRK, J. J. LES of impinging jet flows relevant to vertical landing aircraft, AIAA2005-5226, 23rd AIAA Applied Aero. Conf., Toronto, Canada, 2005.

[37] SPALART, P. Direct simulation of a turbulent boundary layer up to $\mathrm{Re}_{\theta}=1410$. Journal of Fluid Mechanics, February 187, 1988, pp. 61-98.

[38] GANT S.E. Reliability issues of LES-related approaches in an industrial context, Flow, Turbulence and Combustion, March 84, 2010, pp. 325-335.

[39]Dianat, M., McGuiRk, J. J., Fokeer, S., Spencer, A. LES of unsteady vortex aerodynamics in complex geometry gas-turbine fuel injectors, Proc. of 10th ETMM Conference, Marbella, Spain, 2014.

[40] PoPE, S. B. Turbulent Flows, Cambridge University Press, UK, 2010. 
[41] Celik, I. B., Cehreli, Z. N., Yavuz, I. "Index of resolution quality for Large Eddy Simulations, ASME Jnl. of Fluids Eng., No. 5, 127, 2005, pp. 949-958.

[42] CHOI, H. \& MoIN, P. Grid point requirements for Large Eddy Simulation: Chapman estimates revisited, Physics of Fluids, No. 1 24, 2012, 011702.

[43] ANTONIA, R. A. \& LUXTON, R. E. The response of a turbulent boundary layer to a step change in surface roughness, Part 1: smooth to rough, Jnl. of Fluid Mech., No.4, 48, 1971, pp.721-761.

[44] CARLIER, J \& STANISLAS, M. Experimental study of eddy structures in a turbulent boundary layer using particle image velocimetry, Jnl. of Fluid Mech., 535, 2005.

[45]Bourassa, C. \& Thomas, F. O. An experimental investigation of a highly accelerated turbulent boundary layer, Journal of Fluid Mechanics, September 634, 2009, pp. 359-404. 